Pacific

Journal of

Mathematics

QUASIHOMEOMORPHISMS AND UNIVALENT

HARMONIC MAPPINGS ONTO PUNCTURED BOUNDED CONVEX DOMAINS

ABDALLAH LYZZAIK

Volume $200 \quad$ No. 1

September 2001 


\title{
QUASIHOMEOMORPHISMS AND UNIVALENT HARMONIC MAPPINGS ONTO PUNCTURED BOUNDED CONVEX DOMAINS
}

\author{
ABDALLAH LYZZAIK \\ Dedicated to the memory of Professor Ahmad Shamsuddin
}

\begin{abstract}
This paper deals with univalent harmonic mappings of annuli onto punctured bounded convex domains. Several aspects of these mappings are investigated; in particular, boundary functions, existence and uniquenss questions, and the geometry of their analytic and (co-analytic) parts. The paper also considers univalence criteria for Dirichlet solutions in annuli of boundary functions that are a generalized type of homeomorphisms, called quasihomeomorphisms, on one boundary component and constants on the other.
\end{abstract}

\section{Introduction.}

A harmonic mapping $f$ of a region $\mathcal{D}$ is a complex-valued function of the form $f=h+\bar{g}$, where $h$ and $g$ are analytic functions in $\mathcal{D}$, unique up to an additive constant, that are single-valued if $\mathcal{D}$ is simply connected and possibly mutiple-valued otherwise. We call $h$ and $g$ the analytic and co-analytic parts of $f$, respectively. If $f$ is (locally) injective, then $f$ is called (locally) univalent. Note that every conformal and anti-conformal function is a univalent harmonic mapping. The Jacobian and second complex dilatation of $f$ are given by the functions $J(z)=\left|h^{\prime}(z)\right|^{2}-\left|g^{\prime}(z)\right|^{2}$ and $\omega(z)=g^{\prime}(z) / h^{\prime}(z), z \in \mathcal{D}$, respectively. Note that $\omega$ is either a nonconstant meromorphic function or a (possibly infinite) constant. A result of Lewy [13] states that if $f$ is a locally univalent mapping, then its Jacobian $J$ is never zero; namely, for $z \in \mathcal{D}$, either $J(z)>0$ or $J(z)<0$. In the first case $|\omega(z)|<1$ and $f$ is sense-preserving, and in the second $|\omega(z)|>1$ and $f$ is sense-reversing.

A ring domain is a doubly-connected open subset of the plane. Denote by $\mathbb{A}(\rho, 1)$ the annulus $\{z: \rho<|z|<1\}, 0 \leq \rho<1$. It seems that Nitsche [16] was the first to consider univalent harmonic mappings of $\mathbb{A}(\rho, 1)$ onto $\mathbb{A}(R, 1)$. Indeed, Nitsche observed that, unlike with conformal mappings, $R$ can possibly be zero as with the harmonic mapping

$$
f(z)=\left(z-\rho^{2} / \bar{z}\right) /\left(1-\rho^{2}\right)
$$


which can be easily shown to map $\mathbb{A}(\rho, 1)$ univalently onto the punctured $\operatorname{disc} \mathbb{A}(0,1)$. Later, Nitsche $[\mathbf{1 7}, \S 879]$ posed the following question.

Question (Nitsche). All univalent harmonic mappings from $\mathbb{A}(\rho, 1)$ onto $\mathbb{A}(0,1)$, up to a rotation, are of form (1.1).

A negative answer to this question was given by Hengartner and Schober [9]. In their paper, the authors also investigated existence and uniqueness theorems for univalent harmonic mappings with given dilatations between annuli. Subsequently, Hengartner and Szynal [10] and Bshouty and Hengartner [1] gave a representation for harmonic mappings $f$ defined on an annulus $\mathbb{A}(\rho, 1)$ and constant on the inner circle as follows.

Theorem A. Let $f$ be a harmonic function of $\mathbb{A}(\rho, 1), 0<\rho<1$, that extends continuously across $|z|=\rho$ with $f$ identically $\zeta$ there. Then there exists a constant $c$ and a function $h$ analytic in $\mathbb{A}\left(\rho^{2}, 1\right)$ such that

$$
f(z)=h(z)-h\left(\rho^{2} / \bar{z}\right)+\zeta+2 c \log (|z| / \rho) .
$$

Further, if $f$ extends continuously across $|z|=1$, and $f^{*}$ is the restriction of $f$ on $|z|=1$, then $c=0$ if and only if $\zeta$ equals

$$
\zeta_{0}=\frac{1}{2 \pi} \int_{0}^{2 \pi} f^{*}\left(e^{i t}\right) d t
$$

Using Theorem A, Bshouty and Hengartner [1] proved the following result.

Theorem B. Suppose that the following are true:

(i) $\mathbb{G}$ is a bounded convex domain.

(ii) $f^{*}$ is a sense-preserving homeomorphism between the unit circle and $\partial \mathbb{G}$, and the constant $\zeta_{0} \in \mathbb{G}$ given by Equation (1.3) on $|z|=\rho$.

(iii) $f$ is the Dirichlet solution of $f^{*}$ in $\mathbb{A}(\rho, 1)$.

Then $f: \mathbb{A}(\rho, 1) \rightarrow \mathbb{G} \backslash\left\{\zeta_{0}\right\}$ is a homeomorphism.

The author [14, Theorem 2] observed that Theorem B remains true under the weaker condition $f(\mathbb{A}(\rho, 1)) \subset \mathbb{G}$ rather than the convexity of $\mathbb{G}$.

In this paper, we investigate univalent harmonic mappings of ring domains onto bounded punctured convex domains. Throughout the paper we shall use the following notation: $\mathbb{C}$ for the complex plane, $\widehat{\mathbb{C}}$ for the extended complex plane, $\mathbb{D}$ for the open unit disc $\{z \in \mathbb{C}:|z|<1\}$, $\mathbb{T}$ for the unit circle $\{z \in \mathbb{C}:|z|=1\}, 0<\rho<1, \mathbb{T}_{\rho}$ for the circle $\{z \in \mathbb{C}:|z|=\rho\}, \mathbb{A}(\rho, 1)$ for the annulus $\{z \in \mathbb{C}: \rho<|z|<1\}, \mathbb{G}$ for a bounded convex domain. Also, for a subset $S \subset \mathbb{C}$, we denote by $\partial S$ and $\bar{S}$ the boundary and closure of $S$ in $\mathbb{C}$, respectively.

The paper is organized as follows. In Section 2, we describe the boundary functions, called quasihomeomorphisms, of univalent harmonic mappings 
onto punctured convex domains, and extend Theorem B to sense-preserving quasihomeomorphisms. Section 3 is devoted to investigate the geometry of the analytic parts of univalent harmonic mappings in $\mathbb{A}(\rho, 1)$ onto punctured convex domains. One result of this section asserts that these (analytic parts) have nonvanishing derivatives on $\mathbb{T}_{\rho}$, and that they map $\mathbb{T}_{\rho}$ univalently onto Jordan convex curves. Another concludes that these can be written as univalent close-to-convex functions of homeomorphisms in $\mathbb{A}(\rho, 1)$. In Section 4 , we study univalence criteria for Dirichlet solutions in $\mathbb{A}(\rho, 1)$ of boundary functions that are sense-preserving quasihomeomorphisms between $\mathbb{T}$ and $\partial \mathbb{G}$ and constants on $\mathbb{T}_{\rho}$ - a study which was motivated by Hengartner [2, Problem 15]. In Section 5, we prove a uniqueness result implying that the function $f$ defined by (1.1) is the only univalent harmonic mapping, up to rotation, of $\mathbb{A}(\rho, 1)$ onto $\mathbb{A}(0,1)$ having zero as an average value on $\mathbb{T}$ and with analytic part that extends analytically throughout $\mathbb{D}$. This somehow corrects Nitsche's question above and sheds light on Nitsche's insight in that direction.

\section{Quasihomeomorphisms and Univalent Harmonic Mappings.}

The purpose of this section is to characterize the boundary functions of univalent harmonic mappings, and to extend Theorem B to "quasihomeomorphisms". For this purpose, we need the following definition.

Definition 2.1. Let $f$ be a function of $\mathbb{T}$ into a Jordan curve $C$ of $\mathbb{C}$. We say $f$ is a sense-preserving quasihomeomorphism of $\mathbb{T}$ into $C$ if it is a pointwise limit of a sequence of sense-preserving homeomorphisms of $\mathbb{T}$ onto $C$. If in addition, $f$ is a continuous function onto $C$, then $f$ is called a sense-preserving weak homeomorphism.

The definition is based on Bshouty, Hengartner and Naghibi-Beidokhti [3, Definitions 3.1, 3.2]. Sense-preserving quasihomeomorphisms and sensepreserving weak homeomorphisms are characterized as follows.

Proposition 2.1. Let $f$ be a function of $\mathbb{T}$ into a Jordan curve $C$, and let $F$ be a sense-preserving homeomorphism of $\mathbb{T}$ onto $C$.

(i) If $f$ is a sense-preserving quasihomeomorphism of $\mathbb{T}$ onto $C$, then there is a real-valued nondecreasing function $\varphi$ on $\mathbb{R}$ such that $\varphi(t+2 \pi)=$ $\varphi(t)+2 \pi$ and $f\left(e^{i t}\right)=F\left(e^{i \varphi(t)}\right)$.

(ii) If $f\left(e^{i t}\right)=F\left(e^{i \varphi(t)}\right)$, where $\varphi$ is a real-valued nondecreasing function on $\mathbb{R}$ such that $\varphi(t+2 \pi)=\varphi(t)+2 \pi$, and if $E$ is the countable set of points $e^{i \varphi(t)}$ where $\varphi$ is discontinuous, then $f$ coincides on $\mathbb{T} \backslash$ $E$ with a sense-preserving quasihomeomorphism of $\mathbb{T}$. In this case, $f$ is the pointwise limit in $\mathbb{T} \backslash E$ of a sequence of sense-preserving homeomorphisms $f_{n}\left(e^{i t}\right)=F\left(e^{i \varphi_{n}(t)}\right)$ of $\mathbb{T}$ onto $C$, where each $\varphi_{n}$ is a 
real-valued infinite differentiable function on $\mathbb{R}$ such that $\varphi_{n}(t+2 \pi)=$ $\varphi_{n}(t)+2 \pi$ and $\varphi_{n}^{\prime}(t)$ is always positive.

(iii) $f$ is a sense-preserving weak homeomorphism of $\mathbb{T}$ onto $C$ if and only if there is a real-valued continuous nondecreasing function $\varphi$ on $\mathbb{R}$ such that $\varphi(t+2 \pi)=\varphi(t)+2 \pi$ and $f\left(e^{i t}\right)=F\left(e^{i \varphi(t)}\right)$. In this case, $f$ is the uniform limit of a sequence of sense-preserving homeomorphisms $f_{n}\left(e^{i t}\right)=F\left(e^{i \varphi_{n}(t)}\right)$ of $\mathbb{T}$ onto $C$, where each $\left\{\varphi_{n}\right\}$ is a real-valued infinite differentiable function on $\mathbb{R}$ such that $\varphi_{n}(t+2 \pi)=\varphi_{n}(t)+2 \pi$ and $\varphi_{n}^{\prime}(t)$ is always positive.

Proof. (i) There is a sequence $\left\{f_{n}\right\}$ of sense-preserving homeomorphisms of $\mathbb{T}$ onto $C$ that converges pointwise to $f$. We can write $f_{n}\left(e^{i t}\right)=F\left(e^{i \varphi_{n}(t)}\right)$, where each $\left\{\varphi_{n}\right\}$ is a real-valued increasing function on $\mathbb{R}$ such that $0 \leq$ $\varphi_{n}(0)<2 \pi$ and $\varphi_{n}(t+2 \pi)=\varphi_{n}(t)+2 \pi$. Then, by Helly's selection theorem, there is a real-valued nondecreasing function $\varphi$ on $\mathbb{R}$ such that $\varphi(t+2 \pi)=$ $\varphi(t)+2 \pi$ and $\varphi_{n} \rightarrow \varphi$ pointwise in $\mathbb{R}$. Therefore, $f\left(e^{i t}\right)=F\left(e^{i \varphi(t)}\right)$ and (i) follows.

(ii) The function $\varphi(t)-t$ is bounded, a.e. differentiable, and has period $2 \pi$. For fixed $n=1,2, \ldots$, define the function

$$
\varphi_{n}(t)=t+\frac{1}{2 \pi} \int_{0}^{2 \pi} P(1-1 / n, t-\theta)[\varphi(\theta)-\theta] d \theta,
$$

where $P(r, \theta)$ is the Poisson kernel. Then $\varphi_{n}$ is an infinite differentiable function such that $\varphi_{n}(t+2 \pi)=\varphi_{n}(t)+2 \pi$. Also,

$$
\varphi_{n}^{\prime}(t)=\frac{1}{2 \pi} \int_{0}^{2 \pi} P(1-1 / n, t-\theta) d \varphi(\theta)>\frac{1}{2 n-1}
$$

since $P(1-1 / n, t-\theta)<1 /(2 n-1),-\infty \leq t, \theta \leq \infty$. Denote by $E$ the set of points of $\mathbb{T}$ where $e^{i \varphi(t)}$ is discontinuous; then $E$ is countable since $\varphi$ is a nondecreasing function. But by a Schwarz's theorem, $\varphi_{n} \rightarrow \varphi$ pointwise in the set of continuity of $\varphi$. Therefore, $f_{n} \rightarrow f$ pointwise in $\mathbb{T} \backslash E$.

(iii) If $f$ is a sense-preserving weak homeomorphism of $\mathbb{T}$ onto $C$, then, by (i), $f\left(e^{i t}\right)=F\left(e^{i \varphi(t)}\right)$ where $\varphi(t)$ is a real-valued nondecreasing function on $\mathbb{R}$ such that $\varphi(t+2 \pi)=\varphi(t)+2 \pi$. Since $F: \mathbb{T} \rightarrow C$ is a homeomorphism and $f$ is continuous, $e^{i \varphi(t)}=F^{-1} \circ f\left(e^{i t}\right)$ is also continuous in $\mathbb{R}$. This, together with the nonconstancy of $f$, implies that $\varphi$ is also continuous in $\mathbb{R}$.

Suppose now that $f\left(e^{i t}\right)=F\left(e^{i \varphi(t)}\right)$ where $\varphi$ is a real-valued continuous nondecreasing function on $\mathbb{R}$ such that $\varphi(t+2 \pi)=\varphi(t)+2 \pi$. Define the functions $\varphi_{n}$ as in the proof of (ii), and recall that $\varphi_{n}(t+2 \pi)=\varphi_{n}(t)+2 \pi$ and that $\varphi_{n}^{\prime}$ is always positive. Observe that, since $\varphi$ is continuous, $\varphi_{n} \rightarrow$ $\varphi$ uniformly in $\mathbb{R}$. Hence, with $f_{n}\left(e^{i t}\right)=F\left(e^{i \varphi_{n}(t)}\right)$, each $f_{n}$ is a sensepreserving homeomorphism of $\mathbb{T}$ onto $C$ and $f_{n} \rightarrow f$ uniformly on $\mathbb{T}$. This concludes (iii). 
Let $f$ be a function of $\mathbb{A}(\rho, 1)$ into $\widehat{\mathbb{C}}$, and let $\xi \in \mathbb{T}$. We say that $f$ has the unrestricted limit $a \in \widehat{\mathbb{C}}$ at if

$$
f(z) \rightarrow a \quad z \rightarrow \xi, \quad z \in \mathbb{A}(\rho, 1) ;
$$

by defining $f(\xi)=a$ the function $f$ becomes continuous at $\xi$ as a function in $\mathbb{A}(\rho, 1) \cup\{\xi\}$. We shall use $f(\xi)$ to denote the unrestricted limit whenever it exists, and call the resulting function, on its domain of definition in $\mathbb{T}$, the unrestricted limit function $f$. We also define the cluster set $C(f, \xi)$ of $f$ at $\xi$ as the set of all $b \in \widehat{\mathbb{C}}$ for which there are sequences $\left\{z_{n}\right\}$ such that

$$
z_{n} \in \mathbb{A}(\rho, 1), \quad z_{n} \rightarrow \xi, \quad f\left(z_{n}\right) \rightarrow b \quad \text { as } n \rightarrow \infty .
$$

Moreover, If $F$ is a subset of $\mathbb{T}$, then we define the cluster set $C(f, F)$ of $f$ at $F$ as the set-union of the cluster sets $C(f, \xi)$ for $\xi \in E$.

Sense-preserving quasihomeomorphisms are essential for describing the boundary behaviour of univalent harmonic mappings of ring domains onto bounded convex domains. Suppose $f$ is a univalent harmonic mapping of $\mathbb{A}(\rho, 1)$ onto a ring domain $G \backslash\{\zeta\}, \zeta \in \mathbb{G}$. Then either $\lim _{|z| \uparrow 1} f(z)=\zeta$ and $C\left(f, \mathbb{T}_{\rho}\right)=\partial \mathbb{G}$, or $\lim _{|z| \downarrow \rho} f(z)=\zeta$ and $C(f, \mathbb{T})=\partial \mathbb{G}$. In the first case, $f(1 / z)$ becomes a univalent harmonic mapping of $\mathbb{A}(\rho, 1)$ onto $G \backslash\{\zeta\}$ with $\lim _{|z| \downarrow \rho} f(1 / z)=\zeta$ and $C(f(1 / z), \mathbb{T})=\partial \mathbb{G}$. For our study, this leads us to consider, without loss of generality, only univalent harmonic mappings of $\mathbb{A}(\rho, 1)$ onto ring domains $G \backslash\{\zeta\}, \zeta \in \mathbb{G}$, with $\lim _{|z| \downarrow \rho} f(z)=\zeta$.

Definition 2.2. Denote by $\mathcal{H}_{u}(\rho, \mathbb{G})$ the class of all univalent harmonic mappings $f$ of $\mathbb{A}(\rho, 1)$ onto ring domains $G \backslash\{\zeta\}, \zeta \in \mathbb{G}$, with $f\left(\mathbb{T}_{\rho}\right)=\zeta$.

The boundary behavior of functions $f \in \mathcal{H}_{u}(\rho, \mathbb{G})$ is given as follows.

Theorem 2.1. Let $f \in \mathcal{H}_{u}(\rho, \mathbb{G})$. Then there is a countable set $E \subset \mathbb{T}$ such that the following hold:

(i) For each $e^{i \theta} \in \mathbb{T} \backslash E$, the unrestricted limit $f\left(e^{i \theta}\right)$ exists and belongs to $\partial \mathbb{G}$. Furthermore, $f$ is continuous in $\overline{\mathbb{A}(\rho, 1)} \backslash E$.

(ii) For each $e^{i \theta_{0}} \in E$, the side-limits $\lim _{\theta \uparrow \theta_{0}} f\left(e^{i \theta}\right)$ and $\lim _{\theta \downarrow \theta_{0}} f\left(e^{i \theta}\right)$ exist in $\partial \mathbb{G}$ and are distinct.

(iii) For each $e^{i \theta_{0}} \in E$, the cluster set $C\left(f, e^{i \theta_{0}}\right)$ lies in $\partial \mathbb{G}$ and is the straight-line segment joining the side-limits $\lim _{\theta \uparrow \theta_{0}} f\left(e^{i \theta}\right)$ and $\lim _{\theta \downarrow \theta_{0}} f\left(e^{i \theta}\right)$.

(iv) $\overline{c o}(f(\mathbb{T} \backslash E))=\overline{\mathbb{G}}$.

(v) There is a sense-preserving quasihomeomorphism of $\mathbb{T}$ into $\partial \mathbb{G}$ that coincides with the unrestricted limit function $f$ on $\mathbb{T} \backslash E$.

(vi) $f$ is the Dirichlet solution in $\mathbb{A}(\rho, 1)$ of the function $f^{*}$ defined by the unrestricted limit function of $f$ on $\mathbb{T}$ and the value of $f$ on $\mathbb{T}_{\rho}$. 
The fact that $f^{*}$ is not defined on $E$ in (vi) is insignificant. Indeed, Dirichlet solutions in multiply connected domains coincide whenever their boundary functions coincide almost everywhere.

Proof. Applying [8, Theorem 4.3] to $f$ locally at each $e^{i \theta}$ yields (i), (ii), and (iii) except for the inclusion $C\left(f, e^{i \theta_{0}}\right) \subset \partial \mathbb{G}$ which follows because $f: \mathbb{A}(\rho, 1) \rightarrow \partial \mathbb{G}$ is onto. Also, (vi) follows from the maximum principle.

(iv) Since $\overline{\mathbb{G}}$ is convex and each unrestricted limit $f\left(e^{i \theta}\right)$ belongs to $\partial \mathbb{G}$, $\overline{c o}(f(\mathbb{T} \backslash E)) \subset \overline{\mathbb{G}}$. Let $w \in \partial \mathbb{G}$. Because $f: \mathbb{A}(\rho, 1) \rightarrow \mathbb{G}$ is onto, $w$ belongs to the cluster set of some point $\xi \in \mathbb{T}$. If $\xi \in E$, then $w$ is the unrestricted limit $f(\xi)$. If $\xi \notin E$, then $w$ belongs to the boundary straight-line segment joining the side-limits at $\xi$ of the unrestricted function $f$. Note that these limits belong to $\overline{c o}(f(\mathbb{T} \backslash E))$; consequently $w \in \overline{c o}(f(\mathbb{T} \backslash E))$. In either case $\overline{\mathbb{G}} \subset \overline{c o}(f(\mathbb{T} \backslash E))$, and (iv) follows.

(v) Since $\mathbb{G}$ is a Jordan domain, there is a homeomorphism $F$ of $\overline{\mathbb{D}}$ onto $\overline{\mathbb{G}}$ which maps $\mathbb{D}$ conformally onto $\mathbb{G}$. Let $G(z)=F^{-1} \circ f(z), z \in \mathbb{A}(\rho, 1)$. Observe that $G$ is sense-preserving homeomorphism of $\mathbb{A}(\rho, 1)$ into $\mathbb{D}$ which extends continuously to a mapping, also denoted by $G$, from $\mathbb{A}(\rho, 1) \cup(\mathbb{T} \backslash E)$ to $\mathbb{T}$. Let $I=\left\{t:-\infty<t<\infty, e^{i t} \in \mathbb{T} \backslash E\right\}$. We conclude that there is a continuous nondecreasing function $\varphi$ on $I$ such that $G\left(e^{i t}\right)=e^{i \varphi(t)}, t \in I$, and

$$
\sup \{\varphi(t): t \in I \cap[0,2 \pi)\}-\inf \{\varphi(t): t \in I \cap[0,2 \pi)\} \leq 2 \pi .
$$

Extend $\varphi$ to $(-\infty, \infty)$ by defining $\varphi(\tau)=\inf \{\varphi(t): t \in I \cap[0,2 \pi), t>\tau\}$ if $\tau \in[0,2 \pi) \backslash I$, and by letting $\varphi(t+2 \pi)=\varphi(t)+2 \pi$. It is immediate that the new $\varphi$ is a nondecreasing function on $(-\infty, \infty)$ with period $2 \pi$ that is continuous only on $I$. Using Proposition 2.1(ii), the function $F\left(e^{i \varphi(t)}\right)$ coincides with a sense-preserving quasihomeomorphism of $\mathbb{T}$ into $\partial \mathbb{G}$ on $\mathbb{T} \backslash E$.

Now Let $f^{*}$ be a sense-preserving quasihomeomorphism of $\mathbb{T}$ into $\partial \mathbb{G}$. Throughout the paper we denote by $\widetilde{E}\left(f^{*}\right)$ the set of points $e^{i \theta}$ at which $f^{*}$ is continuous. Our second purpose in this section is to show that if $\overline{\mathbb{G}}$ is the closed convex hull of $f^{*}\left(\widetilde{E}\left(f^{*}\right)\right)$, then $f^{*}$ yields a univalent harmonic mapping of $\mathbb{A}(\rho, 1)$ onto the convex domain $\mathbb{G}$ minus one point. This extends Theorem B to sense-preserving quasihomeomorphisms $f^{*}$ of $\mathbb{T}$ into $\partial \mathbb{G}$.

Theorem 2.2. Suppose that the following are true:

(i) $f^{*}$ is a sense-preserving quasihomeomorphism of $\mathbb{T}$ into $\partial \mathbb{G}$, and the constant $\zeta_{0}$ defined by (1.3) on $\mathbb{T}_{\rho}$.

(ii) $\overline{\operatorname{co}} f^{*}\left(\widetilde{E}\left(f^{*}\right)\right)=\overline{\mathbb{G}}$.

(iii) $f$ is the Dirichlet solution of $f^{*}$ in $\mathbb{A}(\rho, 1)$. 
Then $\zeta_{0} \in \mathbb{G}, f \in \mathcal{H}_{u}(\rho, \mathbb{G})$, and there is an analytic function $h$ of $\mathbb{A}\left(\rho^{2}, 1\right)$ such that

$$
f(z)=h(z)-h\left(\rho^{2} / \bar{z}\right)+\zeta_{0}, \quad(z \in \mathbb{A}(\rho, 1)) .
$$

The proof of the theorem requires two lemmas. Let $f^{*}$ be a sensepreserving quasihomeomorphism of $\mathbb{T}$ into $\partial \mathbb{G}$, and let $F$ be a homeomorphism of $\overline{\mathbb{D}}$ onto $\overline{\mathbb{G}}$ that maps $\mathbb{D}$ conformally onto $\mathbb{G}$. By Proposition 2.1(i), there is a real-valued nondecreasing function $\varphi$ on $\mathbb{R}$ such that $\varphi(\theta+2 \pi)=$ $\varphi(\theta)+2 \pi$ and $f^{*}\left(e^{i \theta}\right)=F\left(e^{i \varphi(\theta)}\right)$. If $E$ is the set of points of discontinuity of $e^{i \varphi(\theta)}$ in $\mathbb{T}$, then Proposition 2.1(ii) yields a sequence $\left\{\varphi_{n}\right\}$ of real-valued infinite-differentiable functions on $\mathbb{R}$ such that $\varphi_{n}(\theta+2 \pi)=\varphi_{n}(\theta)+2 \pi$, $\varphi_{n}^{\prime}(\theta)>0$, and $F\left(e^{i \varphi_{n}(\theta)}\right) \rightarrow f\left(e^{i \theta}\right)$ pointwise on $\mathbb{T} \backslash E$. Let $\left\{r_{n}\right\}, \rho<r_{n} \leq 1$, be a sequence converging to 1 , and let $f_{n}^{*}\left(e^{i \theta}\right)=F\left(r_{n} e^{i \varphi_{n}(\theta)}\right)$. Since $F$ is uniformly continuous on $\overline{\mathbb{D}}$, we conclude that $f_{n}^{*}\left(e^{i \theta}\right) \rightarrow f\left(e^{i \theta}\right)$ pointwise on $\mathbb{T} \backslash E$. Note that since $F$ is a convex univalent function, if $r_{n}<1$ then $f_{n}^{*}$ is an infinite-differentiable sense-preserving homeomorphism of $\mathbb{T}$ onto a convex curve in $\mathbb{G}$, and $\left(f_{n}^{*}\right)^{\prime}\left(e^{i \theta}\right)$ is nonvanishing. Define $f^{*}$ and each $f_{n}^{*}$ on $\mathbb{T}_{\rho}$ by the constants $\zeta_{0}$ and $\zeta_{n}$ respectively, where $\zeta_{0}$ is given by (1.3) and

$$
\zeta_{n}=\frac{1}{2 \pi} \int_{0}^{2 \pi} f_{n}^{*}\left(e^{i t}\right) d t
$$

By the bounded convergence theorem, $\zeta_{n} \rightarrow \zeta_{0}$. Now let $f$ and $f_{n}$ be the solutions of the Dirichlet problems of $f^{*}$ and $f_{n}^{*}$ in $\mathbb{A}(\rho, 1)$ respectively. By Theorem A, we can represent $f$ by (1.2) and write each $f_{n}$ as

$$
f_{n}(z)=h_{n}(z)-h_{n}\left(\rho^{2} / \bar{z}\right)+\zeta_{n}
$$

where $h_{n}$ is analytic in $\mathbb{A}\left(\rho^{2}, 1\right)$. Moreover, Theorem B implies that each $f_{n}: \mathbb{A}(\rho, 1) \rightarrow \mathbb{G} \backslash\left\{\zeta_{n}\right\}$ is a homeomorphism.

Under the above assumptions, we prove the requisite lemmas.

Lemma 2.1. $f_{n} \rightarrow f$ locally uniformly in $\mathbb{A}(\rho, 1)$.

Proof. Let $\Phi$ be a local homeomorphism of $\mathbb{D} \backslash\{ \pm 1\}$ onto $\overline{\mathbb{A}(\rho, 1)}$ that maps $\mathbb{D}$ conformally onto $\mathbb{A}(\rho, 1)$, the upper semi-circle: $|\xi|=1, \Im \xi>0$ onto $\mathbb{T}$, and the lower semi-circle: $|\xi|=1, \Im \xi<0$ onto $\mathbb{T}_{\rho}$. Put $T_{n}^{*}=f_{n}^{*} \circ \Phi$, $T^{*}=f^{*} \circ \Phi, T_{n}=f_{n} \circ \Phi$, and $T=f \circ \Phi$. Note that $T_{n}$ and $T$ are the Dirichlet solutions of $T_{n}^{*}$ and $T^{*}$ in $\mathbb{D}$ respectively, and that $T_{n}^{*} \rightarrow T^{*}$ pointwise a.e. in $\mathbb{T}$ since $\zeta_{n} \rightarrow \zeta_{0}$. Hence, for $\eta=R e^{i \Theta}$, we can write

$$
T_{n}(\eta)=\frac{1}{2 \pi} \int_{0}^{2 \pi} P(R, \tau-\Theta) T_{n}^{*}\left(e^{i \tau}\right) d \tau
$$

and

$$
T(\eta)=\frac{1}{2 \pi} \int_{0}^{2 \pi} P(R, \tau-\Theta) T^{*}\left(e^{i \tau}\right) d \tau
$$


Let $K \subset \mathbb{A}(\rho, 1)$ be a compact disc, and let $\widetilde{K}$ be a connected component of $\Phi^{-1}(K)$. Then $\widetilde{K}$ is also compact with a distance $\sigma>0$ from $\mathbb{T}$. Then, for $\eta \in \widehat{K}$

$$
\left|T_{n}(\eta)-T(\eta)\right| \leq \frac{1}{(1-\sigma) \pi} \int_{0}^{2 \pi}\left|T_{n}^{*}\left(e^{i \tau}\right)-T\left(e^{i \tau}\right)\right| d \tau,
$$

and $T_{n} \rightarrow T$ uniformly on $\widetilde{K}$ by the bounded convergence theorem. It follows at once that $f_{n} \rightarrow f$ uniformly on $K$.

Remark 2.1. The above proof uses only the pointwise convergence a.e. of $T_{n}^{*}$ to $T^{*}$ in $\mathbb{T}$ which follows at once from the the pointwise convergence of $f_{n}^{*}$ to $f^{*}$ in $\widetilde{E}\left(f^{*}\right)$. We conclude that if $f^{*}$ and $f_{n}^{*}, n=1,2, \ldots$, are sensepreserving quasihomeomorphisms of $\mathbb{T}$ into $\partial \mathbb{G}$ such that $f_{n}^{*} \rightarrow f^{*}$ pointwise a.e. in $\mathbb{T}$, then $f_{n} \rightarrow f$ locally uniformly in $\mathbb{A}(\rho, 1)$ where $f$ and each $f_{n}$ are as defined above.

Lemma 2.2. (a) $h_{n} \rightarrow h$ locally uniformly in $\mathbb{A}\left(\rho^{2}, 1\right)$.

(b) For $z \in \mathbb{A}(\rho, 1)$,

$$
f(z)=h(z)-h\left(\rho^{2} / \bar{z}\right)+\zeta_{0}=\zeta_{0}+\sum_{k \neq 0} c_{k}\left(f^{*}\right) \frac{r^{2 k}-\rho^{2 k}}{1-\rho^{2 k}} r^{-k} e^{i k \theta}
$$

where $c_{k}\left(f^{*}\right), k= \pm 1, \pm 2, \ldots$, is the $k$-th Fourier coefficient of $f^{*}$.

Proof. For $z=r e^{i \theta}, \rho^{2}<r<1$, and $n=1,2, \ldots$, we have

$$
h_{n}(z)=\sum_{k=-\infty}^{\infty} a_{k}\left(f_{n}^{*}\right) r^{k} e^{i k \theta}
$$

which, with (2.4), yields

$$
f_{n}(z)=\zeta_{n}+\sum_{k \neq 0} a_{k}\left(f_{n}^{*}\right)\left(r^{k}-\frac{\rho^{2 k}}{r^{k}}\right) e^{i k \theta} .
$$

The uniqueness of the Fourier series of $f_{n}\left(r e^{i \theta}\right)$ gives

$$
a_{k}\left(f_{n}^{*}\right)\left(r^{k}-\frac{\rho^{2 k}}{r^{k}}\right)=\frac{1}{2 \pi} \int_{0}^{2 \pi} f_{n}^{*}\left(r e^{i t}\right) e^{-i k t} d t, \quad(k \neq 0) .
$$

Letting $r \rightarrow 1$, the bounded convergence theorem yields

$$
a_{k}\left(f_{n}^{*}\right)\left(1-\rho^{2 k}\right)=\frac{1}{2 \pi} \int_{0}^{2 \pi} f_{n}^{*}\left(e^{i t}\right) e^{-i k t} d t=c_{k}\left(f_{n}^{*}\right), \quad(k \neq 0) .
$$

Hence

$$
a_{k}\left(f_{n}^{*}\right)=\frac{c_{k}\left(f_{n}^{*}\right)}{1-\rho^{2 k}}, \quad(k \neq 0) .
$$


Substituting this in (2.6) yields

$$
f_{n}(z)=\zeta_{n}+\sum_{k \neq 0} c_{k}\left(f_{n}^{*}\right) \frac{r^{2 k}-\rho^{2 k}}{1-\rho^{2 k}} r^{-k} e^{i k \theta} .
$$

Proceeding likewise for $h$, we conclude that if

$$
h(z)=\sum_{k=-\infty}^{\infty} a_{k}\left(f^{*}\right) r^{k} e^{i k \theta},
$$

then

$$
a_{k}\left(f^{*}\right)=\frac{c_{k}\left(f^{*}\right)}{1-\rho^{2 k}}, \quad(k \neq 0) .
$$

Now since $f_{n}^{*}\left(e^{i t}\right) \rightarrow f^{*}\left(e^{i t}\right)$ pointwise in $\mathbb{T} \backslash E, c_{k}\left(f_{n}^{*}\right) \rightarrow c_{k}\left(f^{*}\right)$ uniformly relative to $k$ as $n \rightarrow \infty$. It follows, by (2.8) and (2.7), that $a_{k}\left(f_{n}^{*}\right) \rightarrow a_{k}\left(f^{*}\right)$ uniformly relative to $k$ as $n \rightarrow \infty$. This proves (a). Now since $h_{n}(z) \rightarrow h(z)$ and $f_{n}(z) \rightarrow f(z)$ uniformly in $\mathbb{A}(\rho, 1)$, and $\zeta_{n} \rightarrow \zeta$, we conclude (2.5) by taking the limits of both sides in (2.4).

Proof of Theorem 2.2. First, we show that $\zeta_{0} \in \mathbb{G}$. Obviously, $\zeta_{0} \in \overline{\mathbb{G}}$. Suppose that $\zeta_{0} \in \partial \mathbb{G}$. Since $\overline{\mathbb{G}}$ is convex, there is a real $\theta_{0}$ such that

$$
\Re\left\{e^{i \theta_{0}}\left[f^{*}\left(e^{i \theta}\right)-\zeta_{0}\right]\right\} \geq 0, \quad(0 \leq \theta \leq 2 \pi) .
$$

By virtue of (ii), we conclude that this inequality must be strict in some open interval $(\alpha, \beta)$, where $0 \leq \alpha<\beta \leq 2 \pi$. This implies that

$$
\frac{1}{2 \pi} \int_{0}^{2 \pi} \Re\left\{e^{i \theta_{0}}\left[f^{*}\left(e^{i \theta}\right)-\zeta_{0}\right]\right\} d \theta>0,
$$

and consequently

$$
\frac{1}{2 \pi} \int_{0}^{2 \pi} e^{i \theta_{0}}\left[f^{*}\left(e^{i \theta}\right)-\zeta_{0}\right] d \theta \neq 0
$$

This yields at once

$$
\zeta_{0} \neq \frac{1}{2 \pi} \int_{0}^{2 \pi} f^{*}\left(e^{i \theta}\right) d \theta
$$

which gives a contradiction. Hence, $\zeta_{0} \in \mathbb{G}$.

In view of Lemma $2.2(\mathrm{~b})$, it remains to show that $f: \mathbb{A}(\rho, 1) \rightarrow \mathbb{G} \backslash\left\{\zeta_{0}\right\}$ is a homeomorphism.

We show that $f$ is univalent. Let $f_{n}^{*}$ and $f_{n}, n=1,2, \ldots$, be the functions defined in the first paragraph succeeding the statement of the theorem but with each $r_{n}=1$. Using (2.4), the Jacobian of $f_{n}$ can be written as

$$
J_{n}(z)=\left[\left|z h_{n}^{\prime}(z)\right|^{2}-\left|\rho^{2} h_{n}^{\prime}\left(\rho^{2} / \bar{z}\right)\right|^{2}\right] /|z|^{2}>0, \quad z \in \mathbb{A}(\rho, 1) .
$$

Since $f_{n}$ is univalent and sense-preserving, Lewy's theorem [13] implies $J_{n}(z)>0$; so $h_{n}^{\prime}(z) \neq 0$ for $z \in \mathbb{A}(\rho, 1)$. But, by Lemma $2.2(\mathrm{a}), h_{n}^{\prime} \rightarrow h^{\prime}$ 
locally uniformly in $\mathbb{A}(\rho, 1)$. This implies, by Hurwitz's theorem, that either $h^{\prime}$ is nonvanishing or is identically zero in $\mathbb{A}(\rho, 1)$. If the latter case holds, then $f$ is constant. This yields, by [21, Theorem IV.3] and (ii), that $\partial \mathbb{G}$ is a singleton which contradicts (ii). Hence $h^{\prime}(z) \neq 0$ for $z \in \mathbb{A}(\rho, 1)$. Now the Jacobian of $f$ is given by

$$
J(z)=\left[\left|z h^{\prime}(z)\right|^{2}-\left|\rho^{2} h^{\prime}\left(\rho^{2} / \bar{z}\right)\right|^{2}\right] /|z|^{2}, \quad z \in \mathbb{A}(\rho, 1) .
$$

For $z \in \mathbb{A}(\rho, 1), J(z) \geq 0$ since $J_{n}(z) \rightarrow J(z)$. If $J(z)=0$ for some $z$, then $J$ is identically zero; this follows by applying the maximum principle to the dilatation of $f$ given by

$$
\omega(z)=\frac{\overline{\left(\rho^{2} / \bar{z}\right) h^{\prime}\left(\rho^{2} / \bar{z}\right)}}{z h^{\prime}(z)}, \quad(z \in \mathbb{A}(\rho, 1)) .
$$

This implies, by $[\mathbf{1 4}$, Lemma 2], that $f$ maps $\mathbb{A}(\rho, 1)$ into a straight-line $L$. It follows that the unrestricted limits of $f$ lie in $L$. By [21, Theorem IV.3] and (ii), $f^{*}\left(\widetilde{E}\left(f^{*}\right)\right) \subset L$ which yields $\partial \mathbb{G} \subset L$. This gives a contradiction. Hence $J(z)>0$ for $z \in \mathbb{A}(\rho, 1)$, and Lewy's theorem [13] yields $f$ locally univalent. Now the univalence of $f_{n}$, together with Lemma 2.1, yields $f$ univalent in $\mathbb{A}(\rho, 1)$.

Next, we show that $f: \mathbb{A}(\rho, 1) \rightarrow \mathbb{G} \backslash\left\{\zeta_{0}\right\}$ is onto. Let $\xi \in \mathbb{T}$. The cluster set $C(f, \xi)$ of $f$ at $\xi$ is the singleton $f(\xi) \in \partial \mathbb{G}$ if $f$ has an unrestricted limit at $\xi$, or the straight-line segment $\ell$ joining the points $\lim _{\theta \uparrow \theta_{0}} f\left(e^{i \theta}\right)$ and $\lim _{\theta \downarrow \theta_{0}} f\left(e^{i \theta}\right)$, where $\xi=e^{i \theta_{0}}$, which belong to $\partial \mathbb{G}$ by (ii). Suppose that the latter case holds. If $\ell \not \subset \partial \mathbb{G}$, then $\ell$ is a crosscut of $\mathbb{G}$ which separates $\mathbb{G}$ into two Jordan domains of which one contains $f(\mathbb{A}(\rho, 1))$. If $L$ is the straight-line containing $\ell$, then the cluster set $C(f, \mathbb{T})$ of $f$ on $\mathbb{T}$ lies completely in the closed half-plane bounded by $L$ and containing $f(\mathbb{A}(\rho, 1))$. Consequently, $\overline{c o} f^{*}\left(\widetilde{E}\left(f^{*}\right)\right)$ is a proper subset of $\overline{\mathbb{G}}$ which contradicts (ii). Hence, $\ell \subset \partial \mathbb{G}$, and $C(f, \xi) \subset \mathbb{T}$ for every $\xi \in \mathbb{T}$. Now if $f: \mathbb{A}(\rho, 1) \rightarrow \mathbb{G} \backslash\left\{\zeta_{0}\right\}$ is not onto, then there is $\xi \in \mathbb{T}$ such that $C(f, \xi) \cap \mathbb{G} \neq \varnothing$ which yields a contradiction. Therefore, $f: \mathbb{A}(\rho, 1) \rightarrow \mathbb{G} \backslash\left\{\zeta_{0}\right\}$ is a homeomorphism. This completes the proof.

Remark 2.2. The last paragraph of the above proof is indeed a proof for the following result: Let $f$ be the Dirichlet solution in $\mathbb{A}(\rho, 1)$ of a boundary function $f^{*}$ defined on $\mathbb{T}$ by a sense-preserving quasihomeomorphism into $\partial \mathbb{G}$ with $\overline{c o} f^{*}\left(\widetilde{E}\left(f^{*}\right)\right)=\overline{\mathbb{G}}$, and on $\mathbb{T}_{\rho}$ by a constant $\zeta \in \mathbb{G}$. If $f$ is univalent, then $f: \mathbb{A}(\rho, 1) \rightarrow G \backslash\{\zeta\}$ is a homeomorphism.

Theorems 2.1 and 2.2 provide an interesting relationship between sensepreserving quasihomeomorphisms of $\mathbb{T}$ into $\partial \mathbb{G}$ and univalent harmonic mapping of $\mathbb{A}(\rho, 1)$ onto once punctured $\mathbb{G}$. View two sense-preserving quasihomeomorphisms $f^{*}$ of $\mathbb{T}$ into $\partial \mathbb{G}$ equivalent if they coincide almost everywhere. Let $f^{*}$ and $k^{*}$ be sense-preserving quasihomeomorphisms of $\mathbb{T}$ into 
$\partial \mathbb{G}$. Using Proposition 2.1(i), it is easily seen that $f^{*}$ and $k^{*}$ are equivalent if and only if $\widetilde{E}\left(f^{*}\right)=\widetilde{E}\left(k^{*}\right)$ and $f^{*}$ and $k^{*}$ are identical on $\widetilde{E}\left(f^{*}\right)$. Denote by $\mathcal{Q}(\mathbb{G})$ the class of all (equivalence classes of) sense-preserving quasihomeomorphisms $f^{*}$ of $\mathbb{T}$ into $\partial \mathbb{G}$ satisfying (ii) of Theorem 2.2. It is immediate that if $f^{*} \in \mathcal{Q}(\mathbb{G})$ and $k^{*}$ is equivalent to $f^{*}$, then $k^{*} \in \mathcal{Q}(\mathbb{G})$.

Definition 2.3. Denote by $\mathcal{H}_{0}(\rho, \mathbb{G})$ be the class of all Dirichlet solutions $f$ satisfying (i), (ii) and (iii) of Theorem 2.2.

The classes $\mathcal{Q}(\mathbb{G})$ and $\mathcal{H}_{0}(\rho, \mathbb{G})$ are related as follows.

Theorem 2.3. Define $T: \mathcal{Q}(\mathbb{G}) \rightarrow \mathcal{H}_{0}(\rho, \mathbb{G})$ by $T\left(f^{*}\right)=f$, where $f$ is the Dirichlet solution in $\mathbb{A}(\rho, 1)$ of the boundary function which is $f^{*}$ on $\mathbb{T}$ and the average of $f^{*}$ on $\mathbb{T}_{\rho}$. Then $T$ is bijective. Furthermore, for a sequence $\left\{f_{n}^{*}\right\}$ in $\mathcal{Q}(\mathbb{G})$, the following statements are equivalent:

(a) $f_{n}^{*} \rightarrow f^{*}$ a.e..

(b) $f_{n}^{*} \rightarrow f^{*}$ in $L^{1}$.

(c) $f_{n} \rightarrow f$ locally uniformly in $\mathbb{A}(\rho, 1)$.

Proof. Suppose that for $f_{1}^{*}, f_{2}^{*} \in \mathcal{Q}(\mathbb{G}), T\left(f_{1}^{*}\right)=f_{1}, T\left(f_{2}^{*}\right)=f_{2}$, and $f_{1}=$ $f_{2}$. Then, by Theorem 2.1 (i), $f_{1}^{*}=f_{2}^{*}$ everywhere except possibly on a countable set. This makes $T$ injective. Also, by Theorem 2.1, $T$ is surjective. Hence $T$ is bijective.

The implication (a) $\Rightarrow(\mathrm{b})$ follows by the bounded convergence theorem. Conversely, by Proposition 2.1 (i) and Helly's selection theorem, there is a subsequence $\left\{n_{j}\right\}$ of positive integers such that the sequence $\left\{f_{n_{j}}^{*}\right\}$ converges pointwise to a bounded function $k^{*}$. So, $\left|f_{n_{j}}^{*}-f^{*}\right| \rightarrow\left|k^{*}-f^{*}\right|$ pointwise. Then, by (b) and the bounded convergence theorem, $\int_{0}^{2 \pi}\left|k^{*}-f^{*}\right|=0$. This gives (a), and we conclude (a) $\Leftrightarrow(\mathrm{b})$. On the other hand, by Remark 2.1, (a) $\Rightarrow$ (c). It remains to show (c) $\Rightarrow$ (a). Using Theorem 2.1 (i), there exist sense-preserving quasihomeomorphisms $f^{*}$ and $f_{n}^{*}$ of $\mathbb{T}$ into $\mathbb{G}$ that coincide with the boundary functions of $f$ and $f_{n}$ everywhere except possibly on countable sets, respectively. By Helly's selection theorem, every subsequence of $\left\{f_{n}^{*}\right\}$ contains a subsequence $\left\{f_{n_{j}}^{*}\right\}$ that converges pointwise to some bounded function $k^{*}$. Denote by $k$ the Dirichlet solution in $\mathbb{A}(\rho, 1)$ of the boundary function defined on $\mathbb{T}$ by $k^{*}$ and on $\mathbb{T}_{\rho}$ by the average of $k^{*}$ on $\mathbb{T}$. Then, by Remark $2.1, f_{n_{j}} \rightarrow k$ locally uniformly on $\mathbb{A}(\rho, 1)$. Hence $k=f$. This implies $\widetilde{E}\left(f^{*}\right)=\widetilde{E}\left(k^{*}\right)$ and $f^{*}\left(e^{i \theta}\right)=k^{*}\left(e^{i \theta}\right)$ for $e^{i \theta} \in \widetilde{E}\left(f^{*}\right)$. Hence $f_{n_{j}}^{*} \rightarrow f^{*}$ pointwise a.e.. It follows that $f_{n}^{*} \rightarrow f^{*}$ pointwise a.e. and (c) $\Rightarrow(\mathrm{a})$. This ends the proof. 


\section{Geometry of Analytic Parts of Univalent Harmonic Mappings onto Punctured Convex Domains}

Let $h$ be the analytic part of $f \in \mathcal{H}_{u}(\rho, \mathbb{G})$. The purpose of this section is two-fold: First, to show that $h$ has a nonvanishing derivative on $\mathbb{T}_{\rho}$, and that it maps $\mathbb{T}_{\rho}$ homeomorphically onto a sense-preserving convex Jordan curve whose diameter admits a universal upper bound, and second, to prove that $h$ is a composition of a univalent close-to-convex function and a homeomorphism of $\mathbb{A}(\rho, 1) \cup \mathbb{T}$ onto a ring subdomain of $\bar{D}$ that maps $\mathbb{T}$ homeomorphically onto itself.

Our first result in this section relates univalent harmonic maps in $\mathcal{H}_{u}(\rho, \mathbb{G})$ to their average associates in $\mathcal{H}_{0}(\rho, \mathbb{G})$.

Proposition 3.1. Suppose that the following are true:

(i) $f^{*}$ is a sense-preserving quasihomeomorphism of $\mathbb{T}$ into $\partial \mathbb{G}$ such that $\overline{c o}\left(f^{*}\left(\widehat{E}\left(f^{*}\right)\right)\right)=\overline{\mathbb{G}}$.

(ii) $f$ is the Dirichlet solution in $\mathbb{A}(\rho, 1)$ of the function defined by $f^{*}$ on $\mathbb{T}$ and a constant $\zeta \in \mathbb{G}$ on $\mathbb{T}_{\rho}$.

(iii) $f_{0}$ is the Dirichlet solution of the function defined by $f^{*}$ on $\mathbb{T}$ and the average $\zeta_{0}$ of $f^{*}$ on $\mathbb{T}$.

Then there is an analytic function $h$ in $\mathbb{A}\left(\rho^{2}, 1\right)$ such that

$$
\begin{aligned}
f(z) & =h(z)-h\left(\rho^{2} / \bar{z}\right)+\zeta+2 c_{\zeta} \log (|z| / \rho) \\
& =f_{0}(z)+2 c_{\zeta} \log |z|,
\end{aligned}
$$

where $c_{\zeta}$ is given by (5.4).

Proof. By Theorem A, there is a constant $c$ and an analytic function $h$ of $\mathbb{A}\left(\rho^{2}, 1\right)$ such that

$$
f(z)=h(z)-h\left(\rho^{2} / \bar{z}\right)+\zeta+2 c \log (|z| / \rho), \quad(z \in \mathbb{A}(\rho, 1)) .
$$

By Theorem 2.2, there is an analytic function $h_{0}$ of $\mathbb{A}\left(\rho^{2}, 1\right)$ such that

$$
f_{0}(z)=h_{0}(z)-h_{0}\left(\rho^{2} / \bar{z}\right)+\zeta_{0}, \quad(z \in \mathbb{A}(\rho, 1)) .
$$

Then

$$
\begin{aligned}
\left(f-f_{0}\right)(z)= & \left(h-h_{0}\right)(z)-\left(h-h_{0}\right)\left(\rho^{2} / \bar{z}\right) \\
& +\zeta-\zeta_{0}+2 c \log (|z| / \rho)
\end{aligned}
$$

is a bounded harmonic mapping in $\mathbb{A}(\rho, 1)$. We conclude, by Schwarz's theorem, that the unrestricted limit function of $\left(f-f_{0}\right)$ exists everywhere on $\mathbb{T}$ except possibly on a countable subset $E$. Furthermore, it is identically zero on $\mathbb{T} \backslash E$ by the definition of $f$ and $f_{0}$. Since $\left(f-f_{0}\right)\left(\mathbb{T}_{\rho}\right)=\zeta-\zeta_{0}$, the maximum principle yields

$$
\left(f-f_{0}\right)(z)=\zeta-\zeta_{0}+2 c_{\zeta} \log (|z| / \rho)
$$


where $c_{\zeta}$ is as given in (5.4). By comparing (3.3) and (3.4), it follows that $c=c_{\zeta}$ and $h-h_{0}$ is constant. This yields (3.1) and (3.2), and the proof is complete.

Note that Proposition 3.1 does not require $f$ to be in $\mathcal{H}_{u}(\rho, \mathbb{G})$. If this however is the case, then we obtain Corollary 3.1.

Corollary 3.1. Let $f_{0}$ be the average associate of $f \in \mathcal{H}_{u}(\rho, \mathbb{G})$ with $f\left(\mathbb{T}_{\rho}\right)$ $=\zeta$ and $f_{0}\left(\mathbb{T}_{\rho}\right)=\zeta_{0}$. Then there is an analytic function $h$ in $\mathbb{A}\left(\rho^{2}, 1\right)$ such that (3.1) and (3.2) hold simultaneously.

Suppose now that $f \in \mathcal{H}_{u}(\rho, \mathbb{G})$ has form (1.2). According to Proposition 3.1, $f$ and its average associate $f_{0}$ have the same analytic and coanalytic part $h$. Since our interest in this section is exclusively in $h$, we restrict ourselves to functions $f \in \mathcal{H}_{0}(\rho, \mathbb{G})$ of form $(2.2)$.

We shall need the notion of the module $M(R)$ of a ring domain $R$ [18]. It is known that $R$ is conformally equivalent to a unique annulus $A(r, 1)$, $0<r<1$. In this case $M(R)$ is defined by $\log (1 / r)$ if $r \neq 0$ and by $\infty$ if $r=0$. It is immediate that $M$ is a conformal invariant, and that if $R \subset R^{\prime}$ where $R^{\prime}$ is also a ring domain, then $M(R) \leq M\left(R^{\prime}\right)$ with equality if and only if $R=R^{\prime}$. The Grötzsch's ring domain, $B(t), 0<t<1$, of $R$ is the doubly-connected open subset of $\mathbb{D}$ whose boundary components are $\mathbb{T}$ and the segment $\{x: 0 \leq x \leq t\}$. Observe that $B(t)$ is unique. The module of $B(t)$ is usually denoted by $\mu(t)$. It follows that if $B(s)$ is the Grötzsch's ring domain of $\mathbb{A}(\rho, 1)$, then $\mu(s)=\log (1 / \rho)$. It is known that $\mu$ is a strictly decreasing function of $[0,1)$.

Let $S$ be a subset of $\mathbb{C}$. The diameter of $S$ is the least upper bound of the distances between any two points of $S$. If $\ell_{\alpha}, \alpha \in \mathbb{R}$, is a straight-line in the direction of $e^{i \alpha}$ perpendicular to two support lines $\pi$ and $\pi^{\prime}$ of $S$, then we call the distance between $\ell_{\alpha} \cap \pi$ and $\ell_{\alpha} \cap \pi^{\prime}$ the width of $S$ in the direction of $e^{i \alpha}$. It is known that if $S$ is compact, then the diameter of $S$ is equal to its maximum width [6, p. 77]. In what follows, we denote by $d$ the diameter of $\mathbb{G}$ and by $d_{\alpha}$ its diameter in the direction of $e^{i \alpha}$. We call a Jordan curve convex if it is the boundary of a bounded convex domain.

Using these notions, our result states as follows.

Theorem 3.1. Suppose $f \in \mathcal{H}_{0}(\rho, \mathbb{G})$ has form (2.2). Then

(a) $h^{\prime}$ is nonvanishing on $\mathbb{T}_{\rho}$ and $h$ maps $\mathbb{T}_{\rho}$ homeomorphically onto a convex curve whose diameter is bounded above by

$$
D=(4 d / \pi) \tanh ^{-1}\left(\mu^{-1}(\log (1 / \rho))\right) .
$$

(b) If $h(z)=\sum_{-\infty}^{\infty} a_{n} z^{n}, z \in \mathbb{A}\left(\rho^{2}, 1\right)$, then

$$
\sum_{n=1}^{\infty} n\left|a_{-n}\right|^{2} \rho^{-2 n}<\sum_{n=1}^{\infty} n\left|a_{n}\right|^{2} \rho^{2 n} \leq D^{2} / 4+\sum_{n=1}^{\infty} n\left|a_{-n}\right|^{2} \rho^{-2 n} .
$$


The proof of the theorem needs two lemmas. The first is due to Bshouty and Hengartner [1, Theorem 2.5]. To state this result, we call a ring domain $\Omega$ a slit domain convex in the direction of the real axis if it is obtained by removing a horizontal slit from a domain convex in the direction of the real axis.

Lemma 3.1. Suppose $f \in \mathcal{H}_{u}(\rho, \mathbb{G})$ has form (2.2), and let

$$
\Phi_{\alpha}(z)=e^{i \alpha} h(z)+e^{-i \alpha} \overline{h\left(\rho^{2} / \bar{z}\right)}, \quad\left(z \in \mathbb{A}\left(\rho^{2}, 1\right)\right) .
$$

Then $\Phi_{\alpha}$ is univalent in $\mathbb{A}(\rho, 1)$ and it maps $\mathbb{A}(\rho, 1)$ onto a slit domain convex in the direction of the real axis.

Our second lemma is intuitive and geometric in nature, and it needs some basic notions. A closed curve is a continuous image of $\mathbb{T}$; we use the same notation for the curve and its defining function. Let $\gamma$ be a closed curve, and let $\ell$ be a straight line. A point $w \in \gamma \cap \ell$ is called a meeting point of $\gamma$ and $\ell$ of multiplicity $n$ if $\left|\gamma^{-1}(w)\right|=n$. For a meeting point $w$ of $\gamma$ and $\ell$, we call $w$ a crossing point of $\gamma$ and $\ell$ if there is an open subarc $I$ of $\mathbb{T}$ such that $\gamma^{-1}(w) \cap \ell$ is a singleton and $\ell$ separates $\gamma(I) \backslash\{w\}$.

Lemma 3.2. If every straight-line through the origin meets a closed curve $\gamma$ exactly twice, counting multiplicity, and at crossing points only, then $\gamma$ is a Jordan curve whose inner domain is starlike with respect to the origin.

Proof. We show first that $\gamma$ is a Jordan curve. Suppose that there are points $z_{1}, z_{2} \in \mathbb{T}$ such that $\gamma\left(z_{1}\right)=\gamma\left(z_{2}\right)=w$. If $w=0$, then any straight-line passing through the origin and some other point of $\gamma$ meets $\gamma$ in at least three points, counting multiplicity. If $w \neq 0$, for convenience $w>0$, then $\gamma$ does not meet the negative real axis. This implies, by the compactness of $\gamma$, that $\gamma$ lies within a minimal sector vertexed at the origin whose sides meet $\gamma$ without crossing. In either case, we have a contradiction and the claim holds.

Next, we show that the winding number $n(\gamma, 0)$ is \pm 1 . We consider two cases.

(i) $0 \in \gamma$ : In this case $\gamma$ meets only one of the positive and negative real axes.

(ii) $0 \notin \gamma$ : In this case $\gamma$ meets $\mathbb{R}$ in two distinct points $a$ and $b$, say $a<b$. Here also we consider two cases.

(a) $0<a<b$ or $a<b<0$ : In the first case $\gamma$ does not meet the negative real axis, and in the second it does not meet the positive real axis.

(b) $a<0<b$.

In (i) and (ii.a), the above compactness argument yield a contradiction. Thus only (ii.b) holds. It is immediate then that $n(\gamma, x)=0$ for all $x \in$ $(-\infty, a) \cup(b, \infty)$. Because $\mathbb{R} \cap \gamma=\{a, b\}$ for all $a<x<b$, either $n(\gamma, x) \neq 0$ or $n(\gamma, x)=0$. In the latter case $\gamma \backslash\{a, b\}$ lies completely in one of the upperor lower-half planes and $\mathbb{R}$ fails to cross $\gamma$ at $a$ or $b$. Hence $|n(\gamma, 0)|=1$. 
We further conclude that any straight-line passing through the origin meets the inner domain of $\gamma$ in an open segment containing the origin. Therefore, the inner domain of $\gamma$ is starlike with respect to the origin.

Proof of Theorem 3.1. (a) Fix $\alpha \in \mathbb{R}$ and let $\Phi_{\alpha}$ be given as in (3.5). Then we can write

$$
\Phi_{\alpha}\left(\rho e^{i \theta}\right)=2 \Re\left\{e^{i \alpha} h\left(\rho e^{i \theta}\right)\right\}, \quad(0 \leq \theta \leq 2 \pi) .
$$

Let $m_{\alpha}=\min _{\theta} \Phi_{\alpha}\left(\rho e^{i \theta}\right), M_{\alpha}=\max _{\theta} \Phi_{\alpha}\left(\rho e^{i \theta}\right)$, and $\Gamma$ be the curve defined by $\Gamma(\theta)=h\left(\rho e^{i \theta}\right), 0 \leq \theta \leq 2 \pi$. Observe that $M_{\alpha}-m_{\alpha}$ is the width of $\Gamma$ in the direction of $e^{-i \alpha}$, and that $\Phi_{\alpha}$ maps $\mathbb{T}_{\rho}$ onto the real interval $I_{\alpha}=$ $\left[m_{\alpha}, M_{\alpha}\right]$ which is the inner boundary of the ring domain $\Phi_{\alpha}(\mathbb{A}(\rho, 1))$. Since $\Phi_{\alpha}$ is univalent by Lemma 3.1, $\Phi_{\alpha}^{\prime}$ admits two simple zeros $\rho e^{i \alpha_{1}}$ and $\rho e^{i \alpha_{2}}$, where $\alpha_{1}<\alpha_{2}<\alpha_{1}+2 \pi$, such that $\Phi_{\alpha}\left(\rho e^{i \alpha_{1}}\right)=m_{\alpha}$ and $\Phi_{\alpha}\left(\rho e^{i \alpha_{2}}\right)=M_{\alpha}$. Letting $\Psi(\theta)=\Phi_{\alpha}\left(\rho e^{i \theta}\right), 0 \leq \theta \leq 2 \pi$, we obtain

$$
\Psi^{\prime}(\theta)=i \rho e^{i \theta} \Phi_{\alpha}^{\prime}\left(\rho e^{i \theta}\right)=-2 \Im\left\{e^{i \alpha}\left[\rho e^{i \theta} h^{\prime}\left(\rho e^{i \theta}\right)\right]\right\} .
$$

The first equality yields $\Psi^{\prime}\left(\alpha_{1}\right)=\Psi^{\prime}\left(\alpha_{2}\right)=0, \Psi^{\prime}(\theta)>0$ for $\alpha_{1}<\theta<\alpha_{2}$, and $\Psi^{\prime}(\theta)<0$ for $\alpha_{2}<\theta<\alpha_{1}+2 \pi$. Denote by $\gamma$ the curve defined by $\gamma(\theta)=\rho e^{i \theta} h^{\prime}\left(\rho e^{i \theta}\right), 0 \leq \theta \leq 2 \pi$. The second equality implies that the real axis meets the curve $e^{i \alpha} \gamma$ exactly twice, counting multiplicity, and only at crossing points; namely $\rho e^{i \alpha_{1}} h^{\prime}\left(\rho e^{i \alpha_{1}}\right)$ and $\rho e^{i \alpha_{2}} h^{\prime}\left(\rho e^{i \alpha_{2}}\right)$. This means that the line in the direction of $e^{-i \alpha}$ meets $\gamma$ exactly twice and only at crossing points. Since $\alpha$ is arbitrary, this property also holds for all straight-lines passing through origin. Using Lemma 3.2, we conclude that $\gamma$ is a Jordan curve that bounds a starlike domain with respect to the origin. Thus $h^{\prime}$ is nonvanishing on $\mathbb{T}_{\rho}$ and

$$
\frac{d}{d \theta} \arg \rho e^{i \theta} h^{\prime}\left(\rho e^{i \theta}\right)=\Re\left\{1+\rho e^{i \theta} \frac{h^{\prime \prime}\left(\rho e^{i \theta}\right)}{h^{\prime}\left(\rho e^{i \theta}\right)}\right\}
$$

is always either nonpositive or nonnegative. Hence $\Gamma$ is a convex curve as claimed.

Now we show that the diameter of $\Gamma$ is bounded by $D$. With a fixed $\alpha$ again, we can write

$$
\Phi_{\alpha}(z)=e^{i \alpha}\left(f(z)-\zeta_{0}\right)+2 \Re\left\{e^{i \alpha} \overline{h\left(\rho^{2} / \bar{z}\right)}\right\} .
$$

Geometrically, this means that for every $z \in \mathbb{A}(\rho, 1)$ the value $\Phi_{\alpha}(z)$ can be obtained from the point $e^{i \alpha}\left(f(z)-\zeta_{0}\right)$ by a horizontal shift by $2 \Re\left\{e^{i \alpha} \overline{h\left(\rho^{2} / \bar{z}\right)}\right\}$. Recall $d_{\alpha}, d$, and $I_{\alpha}$. We conclude that the ring domain $\Phi_{\alpha}(\mathbb{A}(\rho, 1))$ is properly contained in a horizontal strip of width $d_{\beta}$, $\beta=\pi / 2-\alpha$, and with a slit along $I_{\alpha}$. Let $S_{\alpha}$ and $S$ be the horizontal strips symmetric about $\mathbb{R}$ and of widths $2 d_{\beta}$ and $2 d$ respectively. Obviously, $\Phi_{\alpha}(\mathbb{A}(\rho, 1))$ is a proper subset of $S_{\alpha} \backslash I_{\alpha}, S_{\alpha} \backslash I_{\alpha} \subset S \backslash I_{\alpha}$, and 
$S \backslash I_{\alpha}$ is conformally equivalent to $S \backslash\left[\left(m_{\alpha}-M_{\alpha}\right) / 2,\left(M_{\alpha}-m_{\alpha}\right) / 2\right]$. Observe that the length of the boundary slit of the Grötzsch's domain of $S \backslash\left[\left(m_{\alpha}-M_{\alpha}\right) / 2,\left(M_{\alpha}-m_{\alpha}\right) / 2\right]$ is $\tanh \left[\pi\left(M_{\alpha}-m_{\alpha}\right) /(4 d)\right]$. Then

$$
\begin{aligned}
\log (1 / \rho) & =M(\mathbb{A}(\rho, 1))<M\left(S_{\alpha} \backslash I_{\alpha}\right) \\
& \leq M\left(S \backslash\left[\left(m_{\alpha}-M_{\alpha}\right) / 2,\left(M_{\alpha}-m_{\alpha}\right) / 2\right]\right) \\
& =\mu\left(\tanh \left[\pi\left(M_{\alpha}-m_{\alpha}\right) /(4 d)\right]\right) .
\end{aligned}
$$

Since $\mu$ is a decreasing function, we obtain

$$
\tanh \left[\pi\left(M_{\alpha}-m_{\alpha}\right) /(4 d)\right]<\mu^{-1}(\log (1 / \rho)),
$$

or

$$
M_{\alpha}-m_{\alpha}<\frac{4 d}{\pi} \tanh ^{-1}\left(\mu^{-1}(\log (1 / \rho))\right)=D .
$$

Note that $\alpha$ may be chosen so that $d=M_{\alpha}-m_{\alpha}$. This concludes (a).

(b) Let $\Omega$ be the closed region bounded by the curve $\Gamma$ defined in the proof of (a). We show first that the area $A(\Omega)$ of $\Omega$ is at most $\pi D^{2} / 4$. By [6, Theorem 54], $\Omega$ is contained in a convex region $\Omega^{\prime}$ of constant width $D$ in every direction. Then Cauchy's theorem [6, p. 127] implies that the perimeter of $\Omega^{\prime}$ is $\pi D$. But the area of $\Omega^{\prime}$ is at most $\pi D^{2} / 4$ by the isoperimetric inequality [6, p. 108]. This proves our claim.

On the other hand,

$$
\begin{aligned}
A(\Omega) & =\frac{1}{2 i} \int_{|z|=\rho} \overline{h(z)} h^{\prime}(z) d z \\
& =\frac{1}{2} \int_{0}^{2 \pi}\left\{\sum_{n=-\infty}^{\infty} \bar{a}_{n} \rho^{n} e^{-i n \theta}\right\}\left\{\sum_{n=-\infty}^{\infty} n a_{n} \rho^{n} e^{i n \theta}\right\} d \theta \\
& =\pi\left\{\sum_{n=1}^{\infty} n\left|a_{n}\right|^{2} \rho^{2 n}-\sum_{n=1}^{\infty} n\left|a_{-n}\right|^{2} \rho^{-2 n}\right\} .
\end{aligned}
$$

Therefore,

$$
\sum_{n=1}^{\infty} n\left|a_{n}\right|^{2} \rho^{2 n}-\sum_{n=1}^{\infty} n\left|a_{-n}\right|^{2} \rho^{-2 n}<D^{2} / 4
$$

and (b) follows.

Next, we embark on proving that the analytic part of every harmonic mapping in $\mathcal{H}_{u}(\rho, \mathbb{G})$ is a univalent close-to-convex function of $\mathbb{D}$ precomposed with a homeomorphism of $\mathbb{A}(\rho, 1) \cup \mathbb{T}$ onto a ring subdomain of $\bar{D}$ that maps $\mathbb{T}$ homeomorphically onto itself. As above, it suffices to consider harmonic mappings $f \in \mathcal{H}_{0}(\rho, \mathbb{G})$.

Theorem 3.2. Suppose $f \in \mathcal{H}_{0}(\rho, \mathbb{G})$ has form (2.2). Then there is a univalent close-to-convex function $H$ of $\mathbb{D}$ and a homeomorphism $\phi$ of $\mathbb{A}(\rho, 1) \cup \mathbb{T}$ into $\overline{\mathbb{D}}$ with $\phi(\mathbb{T})=\mathbb{T}$ such that $h=H \circ \phi$. 
Observe that if $f \in \mathcal{H}_{0}(\rho, \mathbb{G})$ is given by $(2.2)$, then the dilatation of $f$ is given by (2.9).

The proof of the theorem needs two lemmas. The first states as follows.

Lemma 3.3. Fix $p, p=2,3, \ldots$ Suppose $f \in \mathcal{H}_{0}(\rho, \mathbb{G})$ has form $(2.2)$ and an unrestricted limit function that satisfies the following properties:

(i) $f$ is a sense-preserving local homeomorphism of $\mathbb{T}$ onto $\partial \mathbb{G}$.

(ii) $f^{(p)}$ exists and is absolutely continuous on $\mathbb{T}$.

(iii) $f^{\prime}$ is nonvanishing on $\mathbb{T}$.

Then

(a) $h$ extends to $\overline{\mathbb{A}\left(\rho^{2}, 1\right)}$ such that $h\left(e^{i \theta}\right)$ and $h\left(\rho^{2} e^{i \theta}\right)$ are continuously $(p-1)$-differentiable with

$$
\lim _{z \rightarrow r e^{i \theta}} h^{(k)}(z)=h^{(k)}\left(r e^{i \theta}\right), \quad\left(z \in \mathbb{A}\left(\rho^{2}, 1\right)\right),
$$

where $r$ is either 1 or $\rho^{2}$.

(b) $h^{\prime}\left(e^{i \theta}\right) \neq 0$ and $h^{\prime}\left(\rho^{2} e^{i \theta}\right) \neq 0$ for all $\theta$.

(c) $\omega$ extends continuously to $\overline{\mathbb{A}(\rho, 1)}$ such that $\omega\left(e^{i \theta}\right) \neq-1$ and $|\omega(z)| \leq 1$ for $z \in \overline{\mathbb{A}(\rho, 1)}$.

Proof. (a) If

$$
h(z)=\sum_{n=-\infty}^{\infty} a_{n} z^{n}, \quad\left(z \in \mathbb{A}\left(\rho^{2}, 1\right)\right),
$$

then for $z=r e^{i \theta}, \rho<r<1$,

$$
f(z)=\zeta_{0}+\sum_{n \neq 0} a_{n}\left[r^{n}-\left(\rho^{2} / r\right)^{n}\right] e^{i n \theta} .
$$

Since $f^{\prime}\left(e^{i \theta}\right)$ exists for all $\theta,[7$, Theorem 55] gives

$$
f\left(e^{i \theta}\right)=\zeta_{0}+\sum_{n \neq 0} c_{n} e^{i n \theta}
$$

where, by the bounded convergence theorem and (3.7),

$$
\begin{aligned}
c_{n} & =\frac{1}{2 \pi} \int_{0}^{2 \pi} f\left(e^{i \theta}\right) e^{-i n \theta} d \theta=\lim _{r \rightarrow 1} \frac{1}{2 \pi} \int_{0}^{2 \pi} f\left(r e^{i \theta}\right) e^{-i n \theta} d \theta \\
& =\lim _{r \rightarrow 1} a_{n}\left[r^{n}-\left(\rho^{2} / r\right)^{n}\right]=a_{n}\left(1-\rho^{2 n}\right) .
\end{aligned}
$$

Using this in (3.8), we obtain

$$
f\left(e^{i \theta}\right)=\zeta_{0}+\sum_{n \neq 0} a_{n}\left(1-\rho^{2 n}\right) e^{i n \theta} .
$$

Since $f^{(p)}$ is absolutely continuous, [7, Theorem 40] yields

$$
a_{n}\left(1-\rho^{2 n}\right)=o\left(|n|^{-(p+1)}\right) \text {. }
$$


This gives for $k=1,2, \ldots, p-1$,

$$
n(n-1) \cdots(n-k+1) a_{n}=o\left(|n|^{-p+k-1}\right)=o\left(|n|^{-2}\right) .
$$

Define, for $r=1$ or $\rho^{2}$,

$$
h\left(r e^{i \theta}\right)=\sum_{n=-\infty}^{\infty} a_{n} r^{n} e^{i n \theta} .
$$

Observe that term by term differentiation of the latter series yields, by (3.9), a uniformly convergent series. Now term by term integration of the resulting series yields $h\left(r e^{i \theta}\right)$ continuously differentiable. Repeating the same procedure with $h^{\prime}\left(r e^{i \theta}\right)$ yields $h\left(r e^{i \theta}\right)$ continuously 2-differentiable. Observe, again because of (3.9), that the same procedure can be repeated $p-1$ times proving $h\left(r e^{i \theta}\right)$ continuously $(p-1)$-differentiable. Using (3.9) once again, together with the uniform convergence of $k$-th, $k=1,2, \ldots, p-1$, derivatives of $h\left(r e^{i \theta}\right)$ and the above Laurent's series of $h(z)$, yields (3.6).

(b) The Jacobian of $f$ is given by

$$
J(z)=\left[\left|z h^{\prime}(z)\right|^{2}-\left|\rho^{2} h^{\prime}\left(\rho^{2} / \bar{z}\right)\right|^{2}\right] /|z|^{2}, \quad(z \in \mathbb{A}(\rho, 1)) .
$$

Since $f$ is univalent in $\mathbb{A}(\rho, 1)$, Lewy's theorem [13] yields $J(z)>0$ for $z \in \mathbb{A}(\rho, 1)$; that is,

$$
\rho^{2}\left|h^{\prime}\left(\rho^{2} / \bar{z}\right)\right|<\left|z h^{\prime}(z)\right|, \quad(z \in \mathbb{A}(\rho, 1)),
$$

which implies $h^{\prime}(z) \neq 0$ in $\mathbb{A}(\rho, 1)$. Using (a), we conclude

$$
\rho^{2}\left|h^{\prime}\left(\rho^{2} e^{i \theta}\right)\right| \leq\left|h^{\prime}\left(e^{i \theta}\right)\right|, \quad(0 \leq \theta \leq 2 \pi) .
$$

We infer that if $h^{\prime}\left(e^{i \theta}\right)=0$ for some $\theta$, then $\rho^{2} e^{2 i \theta} h^{\prime}\left(\rho^{2} e^{i \theta}\right)=0$. Note that

$$
f^{\prime}\left(e^{i \theta}\right)=h^{\prime}\left(e^{i \theta}\right)+\overline{\rho^{2} e^{2 i \theta} h^{\prime}\left(\rho^{2} e^{i \theta}\right)} .
$$

Thus $f^{\prime}\left(e^{i \theta}\right)=0$ which gives a contradiction. Hence $h^{\prime}\left(e^{i \theta}\right) \neq 0$ for all $\theta$. On the other hand, Theorem 3.1 yields $h^{\prime}\left(\rho e^{i \theta}\right) \neq 0$ for all $\theta$. This concludes (b).

(c) It is immediate from (a), (b), and (2.9) that $\omega$ extends continuously to $\overline{\mathbb{A}(\rho, 1)}$. If $\omega\left(e^{i \theta}\right)=-1$ for some $\theta$, then (2.9) and (3.10) give $f^{\prime}\left(e^{i \theta}\right)=0$ which leads to a contradiction. Now since $f$ is univalent, Lewy's theorem [13] implies $|\omega(z)|<1$ for $z \in \mathbb{A}(\rho, 1)$. Using (2.9) once more, with (b), gives $|\omega(z)| \leq 1$ for $z \in \overline{\mathbb{A}(\rho, 1)}$. This completes the proof.

Our second lemma is a weaker form of Theorem 3.2.

Lemma 3.4. Suppose $f \in \mathcal{H}_{0}(\rho, \mathbb{G})$ has form $(2.2), \partial \mathbb{G}$ an analytic curve, and $f\left(e^{i \theta}\right)$ an infinite-differentiable function with a nonvanishing derivative. Let $\Gamma$ be the convex curve defined by $\Gamma(\theta)=h\left(\rho e^{i \theta}\right), 0 \leq \theta \leq 2 \pi$ (see Theorem 3.1(a)). Then $h$ is a sense-preserving homeomorphism of $\mathbb{T}_{\rho}$ onto 
$\Gamma$, and $h=H \circ \phi$ where $H$ is a univalent close-to-convex function of $\mathbb{D}$ and $\phi$ is a homeomorphism of $\mathbb{A}(\rho, 1) \cup \mathbb{T}$ into $\overline{\mathbb{D}}$ with $\phi(\mathbb{T})=\mathbb{T}$.

Proof. From Lemma 3.3, we infer that $h\left(e^{i \theta}\right)$ is infinite-differentiable with a nonvanishing derivative. Using (3.10) and Lemma 3.3(c), we can write

$$
e^{i \theta} h^{\prime}\left(e^{i \theta}\right)=\frac{e^{i \theta} f^{\prime}\left(e^{i \theta}\right)}{1+\omega\left(e^{i \theta}\right)}
$$

Differentiation of both sides yields

$$
\Re\left\{1+e^{i \theta} \frac{h^{\prime \prime}\left(e^{i \theta}\right)}{h^{\prime}\left(e^{i \theta}\right)}\right\}=\Re\left\{1+e^{i \theta} \frac{f^{\prime \prime}\left(e^{i \theta}\right)}{f^{\prime}\left(e^{i \theta}\right)}\right\}-\Re\left\{\frac{e^{i \theta} \omega^{\prime}\left(e^{i \theta}\right)}{1+\omega\left(e^{i \theta}\right)}\right\} .
$$

Denote by $F$ a conformal map of $\mathbb{D}$ onto $\mathbb{G}$. Since $\partial \mathbb{G}$ is an analytic curve, $F$ extends to a conformal map of $\overline{\mathbb{D}}$ onto $\overline{\mathbb{G}}$. Using the bounded convergence theorem and [18, p. 65], we obtain

$$
\Re\left\{1+e^{i \Theta} \frac{F^{\prime \prime}\left(e^{i \Theta}\right)}{F^{\prime}\left(e^{i \Theta}\right)}\right\} \geq 0, \quad(\Theta \in(-\infty, \infty)) .
$$

Observe that we can write $f\left(e^{i \theta}\right)=F\left(e^{i \Theta(\theta)}\right)$ where $\Theta(\theta)$ is an increasing differentiable function of $(-\infty, \infty)$ such that $\Theta(\theta+2 \pi)=\Theta(\theta)+2 \pi$. It is easy to verify that

$$
\Re\left\{1+e^{i \theta} \frac{f^{\prime \prime}\left(e^{i \theta}\right)}{f^{\prime}\left(e^{i \theta}\right)}\right\}=\Theta^{\prime}(\theta) \Re\left\{1+e^{i \Theta} \frac{F^{\prime \prime}\left(e^{i \Theta}\right)}{F^{\prime}\left(e^{i \Theta}\right)}\right\} .
$$

Thus

$$
\int_{0}^{2 \pi} \Re\left\{1+e^{i \theta} \frac{f^{\prime \prime}\left(e^{i \theta}\right)}{f^{\prime}\left(e^{i \theta}\right)}\right\} d \theta=\int_{0}^{2 \pi} \Re\left\{1+e^{i \Theta} \frac{F^{\prime \prime}\left(e^{i \Theta}\right)}{F^{\prime}\left(e^{i \Theta}\right)}\right\} d \Theta=2 \pi
$$

and

$$
\Re\left\{1+e^{i \theta} \frac{f^{\prime \prime}\left(e^{i \theta}\right)}{f^{\prime}\left(e^{i \theta}\right)}\right\} \geq 0, \quad(\theta \in(-\infty, \infty))
$$

since $\Theta^{\prime}(\theta)>0$. On the other hand, by Lemma 3.3(c), $\Re\left[1+\omega\left(e^{i \theta}\right)\right]>0$. Since

$$
\Re\left\{\frac{e^{i \theta} \omega^{\prime}\left(e^{i \theta}\right)}{1+\omega\left(e^{i \theta}\right)}\right\}=\frac{d}{d \theta} \arg \left[1+\omega\left(e^{i \theta}\right)\right]
$$

we conclude

$$
\int_{0}^{2 \pi} \Re\left\{\frac{e^{i \theta} \omega^{\prime}\left(e^{i \theta}\right)}{1+\omega\left(e^{i \theta}\right)}\right\} d \theta=0
$$

and, for $\theta_{1} \leq \theta_{2}<\theta_{1}+2 \pi$,

$$
\int_{\theta_{1}}^{\theta_{2}} \Re\left\{\frac{\omega^{\prime}\left(e^{i \theta}\right)}{1+\omega\left(e^{i \theta}\right)}\right\} d \theta=\arg \left\{\frac{1+\omega\left(e^{i \theta_{2}}\right)}{1+\omega\left(e^{i \theta_{1}}\right)}\right\}>-\pi .
$$


Using (3.12), with (3.15) and (3.17), we get

$$
\int_{0}^{2 \pi} \Re\left\{1+e^{i \theta} \frac{h^{\prime \prime}\left(e^{i \theta}\right)}{h^{\prime}\left(e^{i \theta}\right)}\right\} d \theta=2 \pi,
$$

and, with (3.16) and (3.18), we get

$$
\int_{\theta_{1}}^{\theta_{2}} \Re\left\{1+e^{i \theta} \frac{h^{\prime \prime}\left(e^{i \theta}\right)}{h^{\prime}\left(e^{i \theta}\right)}\right\} d \theta>-\pi, \quad\left(\theta_{1} \leq \theta_{2}<\theta_{1}+2 \pi\right) .
$$

Using Lemma 3.3(b) and (3.19), the argument principle gives

$$
\int_{0}^{2 \pi} \Re\left\{1+\rho e^{i \theta} \frac{h^{\prime \prime}\left(\rho e^{i \theta}\right)}{h^{\prime}\left(\rho e^{i \theta}\right)}\right\} d \theta=2 \pi .
$$

This, together with Theorem 3.1(a), implies

$$
\Re\left\{1+\rho e^{i \theta} \frac{h^{\prime \prime}\left(\rho e^{i \theta}\right)}{h^{\prime}\left(\rho e^{i \theta}\right)}\right\} \geq 0
$$

for all $\theta$, and consequently the convex curve $\Gamma$ is positively-oriented.

Now let $\Omega$ be the convex domain bounded by $\Gamma$. Since $h$ is a sensepreserving homeomorphism of $\mathbb{T}$ onto $\Gamma$, Schoenflies theorem $[\mathbf{1 8}$, p. 25] extends $h$ to a local homeomorphism of $\mathbb{D}$ which maps the closed disc bounded by $\mathbb{T}_{\rho}$ homeomorphically onto $\Omega$. Let $W$ be the image surface of $h$ in $\mathbb{D}$. Note that $W$ is a simply connected hyperbolic covering of $\mathbb{C}$. Hence, by the Uniformization theorem, there is a locally univalent function $H$ of $\mathbb{D}$ with image surface $W$. Define $\phi=H^{-1} \circ h ; \phi$ is obviously a conformal map of $\mathbb{A}(\rho, 1)$ onto a ring subdomain of $\mathbb{D}$ that extends conformally between the unit circles. Write $\phi\left(e^{i \theta}\right)=e^{i \tau}, 0 \leq \theta, \tau \leq 2 \pi$. Observe that $H\left(e^{i \tau}\right)$ is infinite-differentiable with $H^{\prime}\left(e^{i \tau}\right) \neq 0$, since both $h$ and $\phi$ are, and that $\Im\left[e^{i \theta} \phi^{\prime}\left(e^{i \theta}\right) / \phi\left(e^{i \theta}\right)\right]=0$ for all $\theta$. Then direct computation yields

$$
\Re\left\{1+e^{i \tau} \frac{H^{\prime \prime}\left(e^{i \tau}\right)}{H^{\prime}\left(e^{i \tau}\right)}\right\} d \tau=R e\left\{1+e^{i \theta} \frac{h^{\prime \prime}\left(e^{i \theta}\right)}{h^{\prime}\left(e^{i \theta}\right)}\right\} d \theta,
$$

which, with (3.19), gives

$$
\int_{0}^{2 \pi} \Re\left\{1+e^{i \tau} \frac{H^{\prime \prime}\left(e^{i \tau}\right)}{H^{\prime}\left(e^{i \tau}\right)}\right\} d \tau=2 \pi
$$

and, with (3.20), gives

$$
\int_{\theta_{1}}^{\theta_{2}} \Re\left\{1+e^{i \tau} \frac{H^{\prime \prime}\left(e^{i \tau}\right)}{H^{\prime}\left(e^{i \tau}\right)}\right\} d \tau>-\pi, \quad\left(\theta_{1} \leq \theta_{2}<\theta_{1}+2 \pi\right) .
$$

It follows from Kaplan's proof [11, Theorem 2] that $H$ is a univalent closeto-convex function. This completes the proof. 
Proof of Theorem 3.2. Using the ideas in the paragraph succeeding the statement of Theorem 2.2, there exists a sequence $\left\{f_{n}\right\}$ of functions in $\mathcal{H}_{0}(\rho, \mathbb{G})$ with form $(2.4)$ such that each $f_{n}\left(e^{i \theta}\right)$ is an infinite-differentiable function in $\mathbb{T}$, and $f_{n} \rightarrow f$ and $h_{n} \rightarrow h$ locally uniformly in $\mathbb{A}(\rho, 1)$ and $\mathbb{A}\left(\rho^{2}, 1\right)$ respectively. Let $\Gamma$ be the convex curve defined by $\Gamma(\theta)=h\left(\rho e^{i \theta}\right)$, $0 \leq \theta \leq 2 \pi$, and let $\Omega$ be the convex domain bounded by $\Gamma$. Also, let $\Gamma_{n}$ be the convex curve defined by the function $h_{n}\left(\rho e^{i \theta}\right), 0 \leq \theta \leq 2 \pi$. By Lemma 3.4, each $h_{n}$ is a sense-preserving homeomorphism of $\mathbb{T}_{\rho}$ onto $\Gamma_{n}$ with

$$
\Re\left\{1+\rho e^{i \theta} \frac{h_{n}^{\prime \prime}\left(\rho e^{i \theta}\right)}{h_{n}^{\prime}\left(\rho e^{i \theta}\right)}\right\} \geq 0
$$

for all $\theta$; see (3.22). Using this, with Lemma 2.2(a) and Theorem 3.1(a), we conclude that $h$ satisfies (3.22) and, consequently, $h$ is also a sensepreserving homeomorphism of $\mathbb{T}_{\rho}$ onto $\Gamma$. Also, by Lemma 3.4, we have each $h_{n}$ univalent in $\mathbb{A}(\rho, 1)$. Hence, by Hurwitz's theorem, $h$ is also a univalent function on $\mathbb{A}(\rho, 1)$ or else $f$ is a constant. Define $W$ as above, $\Omega_{n}$ as the convex domain bounded by $\Gamma_{n}$, and $W_{n}=h_{n}(\mathbb{A}(\rho, 1)) \cup \overline{\Omega_{n}}$. It is immediate that $W$ and each $W_{n}$ are simply connected domains in $\mathbb{C}$. Fix a point $\varrho \in \Omega$. We show that

$$
W_{n} \rightarrow W \quad \text { as } n \rightarrow \infty
$$

with respect to $\varrho$

in the sense of Carathéodary's kernel convergence [18, pp. 13-15]. Let $w_{0} \in$ $W$. We show first that $w_{0} \in W_{n}$ for sufficiently large $\mathrm{n}$. Let $\gamma$ be a separating Jordan curve in $\mathbb{A}(\rho, 1)$ with $w_{0}$ in the interior domain of $h(\gamma)$. Since $h_{n} \rightarrow h$ uniformly on $\gamma, w_{0}$ belongs to the interior domain of the Jordan curve $h_{n}(\gamma)$ for sufficiently large $n$. Since each $W_{n}$ is simply connected, $w_{0} \in W_{n}$ for sufficiently large $n$. Now let $w_{0} \in \partial W$. We show that $w_{0}$ is the limit point of a sequence $\left\{w_{n}\right\}$ where $w_{n} \in \partial W_{n}$. Suppose that this is false. Then there is an increasing sequence of positive integers $\left\{n_{\nu}\right\}$ and an open neighborhood $V$ of $w_{0}$ such that $\partial W_{n_{\nu}} \cap V=\varnothing$. Also, choose $V$ so that $\bar{\Omega} \cap \bar{V}=\varnothing$; this is possible since $\Gamma_{n} \rightarrow \Gamma$. It follows that, for each $n_{\nu}$, either $V \cap W_{n_{\nu}}=\varnothing$ or $V \subset W_{n_{\nu}}$. Suppose that the first case happens infinitely often, say, without loss of generality, for all $\nu$. Then $h_{n_{\nu}}(z) \notin V$ for $z \in \mathbb{A}(\rho, 1)$. Since $h_{n_{\nu}}(z) \rightarrow h(z), h(\mathbb{A}(\rho, 1)) \bigcap V=\varnothing$ and we have a contradiction. Now suppose, without loss of generality, that $V \subset W_{n_{\nu}}$ for all $\nu$. Then the inverse function $\psi_{n_{\nu}}(w)=h_{n_{\nu}}^{-1}(w)$ is analytic in $V$ with $\left|\psi_{n_{\nu}}(w)\right|<1$. By Montel's theorem, we can find a subsequence of $\left\{\psi_{n_{\nu}}\right\}$ that converges locally uniformly in $V$. Suppose, without loss of generality, that $\left\{\psi_{n_{\nu}}\right\}$ converges locally uniformly in $V$. Then the limit function $\psi$ satisfies $\rho \leq|\psi(w)| \leq 1$ for $w \in V$. By Hurwitz's theorem, either $\psi$ is a constant or is a univalent function in $V$. We show that the latter holds. To do so, we show first that $\left\{\psi_{n_{\nu}}\right\}$ converges locally uniformly in $h(\mathbb{A}(\rho, 1))$ even though these functions may not be defined in $h(\mathbb{A}(\rho, 1))$ in the proper sense. Let 
$\Delta$ be a closed Jordan region in $h(\mathbb{A}(\rho, 1))$, and let $K$ be a compact subset of the interior $\Delta$. Since $h$ is univalent, $h^{-1}(\Delta)$ is a closed Jordan region in $\mathbb{A}(\rho, 1)$ whose interior contains $h^{-1}(K)$. Since $h_{n_{\nu}} \rightarrow h$ uniformly on $h^{-1}(\Delta)$, an argument using Rouche's theorem implies that $K \subset h_{n_{\nu}} \circ h^{-1}(\Delta)$ or $K \subset h_{n_{\nu}}(\mathbb{A}(\rho, 1))$ for sufficiently large $n_{\nu}$. A compactness argument also yields the same conclusion for any compact subset $K$ of $h(\mathbb{A}(\rho, 1))$. So, for a given compact subset $K$ of $h(\mathbb{A}(\rho, 1))$, the functions $\psi_{n_{\nu}}$ are defined on $K$ for sufficiently large $n_{\nu}$. Since the range of each $\psi_{n_{\nu}}$ is $\mathbb{A}(\rho, 1)$, the sequence $\left\{\psi_{n_{\nu}}\right\}$ is a normal family in $h(\mathbb{A}(\rho, 1))$. Since $V \bigcap h(\mathbb{A}(\rho, 1)) \neq$ $\varnothing, \psi_{n_{\nu}} \rightarrow \psi$ in $h(\mathbb{A}(\rho, 1))$. Recall the above curve $\gamma$. If $\psi$ is constant, then $\psi_{n_{\nu}}(h(\gamma))$ admits an arbitrarily small diameter for large $n_{\nu}$ which is impossible since each curve $\psi_{n_{\nu}}(\gamma)$ separates $\mathbb{A}(\rho, 1)$. Hence $\psi$ is univalent in $V$ and $\rho<|\psi(w)|<1$ for $w \in V$, in particular $\psi\left(w_{0}\right) \in \mathbb{A}(\rho, 1)$. It follows that $\left\{h_{n_{\nu}}\right\}$ converges locally uniformly near $\psi\left(w_{0}\right)$. Since $\psi_{n_{\nu}}\left(w_{0}\right) \rightarrow \psi\left(w_{0}\right)$ and $w_{0}=h_{n_{\nu}} \circ \psi_{n_{\nu}}\left(w_{0}\right)$, we conclude $w_{0}=h\left(\psi\left(w_{0}\right)\right)$. This contradicts $w_{0} \in \partial W$ and (3.23) holds.

Now define $H$ as above but with the additional conditions $H(0)=\varrho$ and $H^{\prime}(0)>0$. Also, let $H_{n}$ be the conformal map of $\mathbb{D}$ onto $W_{n}$ satisfying $H_{n}(0)=\varrho$ and $H_{n}{ }^{\prime}(0)>0$. By Carathéodary's kernel theorem [18, pp. 13$15], H_{n} \rightarrow H$ locally uniformly in $\mathbb{D}$. Since, by Lemma 3.4, each $H_{n}$ is a univalent close-to-convex function, $H$ is also a univalent close-to-convex function. Letting $\phi=H^{-1} \circ h$. It is easily seen that $\phi$ satisfies the desired properties. This ends the proof.

\section{Univalent Harmonic Mappings onto Punctured Convex Domains.}

Let $f$ be the Dirichlet solution in $\mathbb{A}(\rho, 1)$ of a function $f^{*}$ of $\partial \mathbb{A}(\rho, 1)$ defined on $\mathbb{T}$ be a sense-preserving quasihomeomorphism into $\partial \mathbb{G}$ satisfying $\overline{c o} f^{*}\left(\widetilde{E}\left(f^{*}\right)\right)=\overline{\mathbb{G}}$, and on $\mathbb{T}_{\rho}$ by a constant $\zeta \in \mathbb{G}$. Theorem 2.2 asserts that $f$ belongs to $\mathcal{H}_{u}(\rho, \mathbb{G})$ if $\zeta=\zeta_{0}$, where $\zeta_{0}$ is the average of $f^{*}$ on $\mathbb{T}_{\rho}$ given by (1.3). Recently however, Duren and Hengartner [5, Example 1] observed that this condition is not necessary, and showed that the harmonic mapping

$$
F(z)=\left(z-\rho^{2} / \bar{z}\right) /\left(1-\rho^{2}\right)+2 c \log |z|, \quad(z \in \mathbb{A}(\rho, 1)),
$$

belongs to $\mathcal{H}_{u}(\rho, \mathbb{D})$ with $f(0)=2 c \log \rho$ if $|c|<\rho /\left(1-\rho^{2}\right)$. Note that the boundary function of $F$ is the identity map on $\mathbb{T}$ and the constant $2 c \log \rho$ on $\mathbb{T}_{\rho}$. In view of this, Hengartner [2, Problem 15] suggested the problem of finding the set of values $\zeta \in \mathbb{G}$ that yields $f: \mathbb{A}(\rho, 1) \rightarrow G \backslash\{\zeta\}$ a homeomorphism.

Now, let $f^{*}$ be a sense-preserving quasihomeomorphism of $\mathbb{T}$ into $\partial \mathbb{G}$ with $\overline{c o} f^{*}\left(\widetilde{E}\left(f^{*}\right)\right)=\overline{\mathbb{G}}$. Denote by $\mathcal{H}\left(\rho, f^{*}\right)$ the class of Dirichlet solutions in $\mathbb{A}(\rho, 1)$ of functions of $\partial \mathbb{A}(\rho, 1)$ defined on $\mathbb{T}$ by $f^{*}$ and on $\mathbb{T}_{\rho}$ by some 
constant $\zeta \in \mathbb{G}$. Also, denote by $\mathcal{H}_{u}\left(\rho, f^{*}\right)$ the subclass of $\mathcal{H}\left(\rho, f^{*}\right)$ of univalent mappings. Of interest shall be the set $K\left(\rho, f^{*}\right)$ of values $\zeta \in \mathbb{G}$ for which a function $f \in \mathcal{H}\left(\rho, f^{*}\right)$ belongs to $\mathcal{H}_{u}\left(\rho, f^{*}\right)$.

Our first result in this section states that $K\left(\rho, f^{*}\right)$ is compact. In view of Proposition 3.1, the class $\mathcal{H}\left(\rho, f^{*}\right)$ yields an analytic function $h$ in $\mathbb{A}\left(\rho^{2}, 1\right)$, unique up to an additive constant, such that every $f \in \mathcal{H}_{u}\left(\rho, f^{*}\right)$ is of the forms (3.1) and (3.2). In our second result, we characterize in terms of $h$ and $f^{*}$ the boundary points of $K\left(\rho, f^{*}\right)$ in a manner leading to a univalence criterion for functions $f \in \mathcal{H}\left(\rho, f^{*}\right)$. Finally, we provide sufficient conditions on $\rho, \mathbb{G}$, and $f^{*}$ that warrant a nonempty interior for $K\left(\rho, f^{*}\right)$.

Theorem 4.1. $K\left(\rho, f^{*}\right)$ is a nonempty compact subset of $\mathbb{G}$.

Proof. Let $\zeta_{0}$ be the average of $f^{*}$ on $\mathbb{T}$. It is immediate from Theorem 2.2 that $\zeta_{0} \in K\left(\rho, f^{*}\right)$. Hence $K\left(\rho, f^{*}\right) \neq \varnothing$.

Suppose that a sequence $\left\{\zeta_{n}\right\}_{n=1}^{\infty}$ in $K\left(\rho, f^{*}\right)$ converges to $\zeta \in \overline{\mathbb{G}}$. We show that $\zeta \in K\left(\rho, f^{*}\right)$. Clearly, there is a unique function $f_{n} \in \mathcal{H}_{u}\left(\rho, f^{*}\right)$ such that $f_{n}\left(\mathbb{T}_{\rho}\right)=\zeta_{n}$. By Proposition 3.1, we can find an analytic function $h$ in $\mathbb{A}\left(\rho^{2}, 1\right)$, unique up to an additive constant, such that

$$
f_{n}(z)=h(z)-h\left(\rho^{2} / \bar{z}\right)+\zeta_{n}+2 c_{n} \log (|z| / \rho), \quad(z \in \mathbb{A}(\rho, 1)),
$$

where $c_{n}=\left(\zeta_{n}-\zeta_{0}\right) /(2 \log \rho)$. Obviously, $c_{n} \rightarrow c=\left(\zeta-\zeta_{0}\right) /(2 \log \rho)$ as $n \rightarrow \infty$. Using $h$ and $c$, we define the harmonic mapping $f$ as in (1.2). If $c=0$, then $\zeta=\zeta_{0} \in K\left(\rho, f^{*}\right)$ by Theorem 2.2. So, suppose that $c \neq 0$. Then $f_{n} \rightarrow f$ (locally) uniformly in $\mathbb{A}(\rho, 1)$. It is easy to see that the Jacobian of $f_{n}$ is given by

$$
J_{n}(z)=\left[\left|z h^{\prime}(z)+c_{n}\right|^{2}-\left|\left(\rho^{2} / \bar{z}\right) h^{\prime}\left(\rho^{2} / \bar{z}\right)+c_{n}\right|^{2}\right] /|z|^{2}, \quad(z \in \mathbb{A}(\rho, 1)) .
$$

Since $f_{n}$ is univalent and sense-preserving, $J_{n}(z)>0$, and consequently $\left|z h^{\prime}(z)+c_{n}\right| \neq 0$ for $z \in \mathbb{A}(\rho, 1)$. But $z h^{\prime}(z)+c_{n} \rightarrow z h^{\prime}(z)+c$ uniformly in $\mathbb{A}(\rho, 1)$. Hence, by Hurwitz's theorem, either $z h^{\prime}(z)+c \neq 0$ or $z h^{\prime}(z)=0$ for $z \in \mathbb{A}(\rho, 1)$. If the latter holds, then $h^{\prime}(z)=-c / z$ which contradicts the analyticity of $h$ in $\mathbb{A}(\rho, 1)$. Hence $z h^{\prime}(z)+c \neq 0$. The Jacobian of $f$ is now given by

$$
J(z)=\left[\left|z h^{\prime}(z)+c\right|^{2}-\left|\left(\rho^{2} / \bar{z}\right) h^{\prime}\left(\rho^{2} / \bar{z}\right)+c\right|^{2}\right] /|z|^{2}, \quad(z \in \mathbb{A}(\rho, 1)) .
$$

Clearly, $J_{n} z \rightarrow J(z)$. Since $J_{n}(z)>0, J(z) \geq 0$. Thus

$$
\left|\left(\rho^{2} / \bar{z}\right) h^{\prime}\left(\rho^{2} / \bar{z}\right)+c\right| \leq\left|z h^{\prime}(z)+c\right|, \quad(z \in \mathbb{A}(\rho, 1)) .
$$

We prove that this inequality must be strict. Suppose that equality holds for some $z$. Then the maximum principle yields that the dilatation of $f$ 
given by

$$
\omega(z)=\frac{\overline{\left(\rho^{2} / \bar{z}\right) h^{\prime}\left(\rho^{2} / \bar{z}\right)}+\bar{c}}{z h^{\prime}(z)+c}, \quad(z \in \mathbb{A}(\rho, 1)),
$$

is a unimodular constant $e^{2 i \alpha}$ for some real $\alpha$. That is,

$$
\overline{\left(\rho^{2} / \bar{z}\right) h^{\prime}\left(\rho^{2} / \bar{z}\right)}+\bar{c}=e^{2 i \alpha}\left(z h^{\prime}(z)+c\right), \quad(z \in \mathbb{A}(\rho, 1)) .
$$

Since $h$ is analytic in $\mathbb{A}\left(\rho^{2}, 1\right)$, (4.5) holds for $z \in \mathbb{A}\left(\rho^{2}, 1\right)$. In particular, for all $\theta$,

$$
\overline{\rho e^{i \theta} h^{\prime}\left(\rho e^{i \theta}\right)+c}=e^{2 i \alpha}\left[\rho e^{i \theta} h^{\prime}\left(\rho e^{i \theta}\right)+c\right] .
$$

This means that the function $z h^{\prime}(z)$ maps $\mathbb{T}_{\rho}$ into the straight-line passing through $-c$ in the direction of $e^{-i \alpha}$. We conclude that $h(z)$ maps $\mathbb{T}_{\rho}$ to a straight-line in the direction of $e^{i}(\pi / 2-\alpha)$. This contradicts Theorem 3.1. Therefore,

$$
\left|\left(\rho^{2} / \bar{z}\right) h^{\prime}\left(\rho^{2} / \bar{z}\right)+c\right|<\left|z h^{\prime}(z)+c\right| \quad(z \in \mathbb{A}(\rho, 1)) .
$$

This yields $J(z)>0$ for $z \in \mathbb{A}(\rho, 1)$, and consequently $f$ is locally univalent function by Lewy's theorem [13]. Since each $f_{n}$ is univalent and $f_{n} \rightarrow f$ uniformly in $\mathbb{A}(\rho, 1), f$ is univalent in $\mathbb{A}(\rho, 1)$. Using this, with the fact $\overline{c o} f^{*}\left(\widetilde{E}\left(f^{*}\right)\right)=\overline{\mathbb{G}}$, we infer, by Remark 2.2 , that $f: \mathbb{A}(\rho, 1) \rightarrow G \backslash\{\zeta\}$ is a homeomorphism. Therefore $\zeta \in K\left(\rho, f^{*}\right)$ and the proof is complete.

Our second result is Theorem 4.2.

Theorem 4.2. Let $f \in \mathcal{H}_{u}\left(\rho, f^{*}\right)$ be of form (3.1), where $f^{*}: \mathbb{T} \rightarrow \partial \mathbb{G}$ is a twice-differentiable function with nonvanishing derivative and absolutely continuous second derivative. Then the dilatation of $f$ and $z h^{\prime}(z)+c_{\zeta}$ extend continuously to $\mathbb{A}(\rho, 1) \cup \mathbb{T}$ such that $e^{i \theta} h^{\prime}\left(e^{i \theta}\right)+c_{\zeta} \neq 0$ for all $\theta$. Moreover, we have:

(a) If $\zeta \in \partial K\left(\rho, f^{*}\right)$, then either $\rho e^{i \theta_{1}} h^{\prime}\left(\rho e^{i \theta_{1}}\right)+c_{\zeta}=0$ for some $\theta_{1}$, or $\left|\omega\left(e^{i \theta_{2}}\right)\right|=1$ for some $\theta_{2}$.

(b) If $\left|\omega\left(e^{i \theta}\right)\right|=1$ for some $\theta$, then $\zeta \in \partial K\left(\rho, f^{*}\right)$.

(c) If in (a) and (b) the function $\left|\omega\left(e^{i \theta}\right)\right|$ is replaced by the function

$$
2 \Re\left\{\frac{e^{i \theta} h^{\prime}\left(e^{i \theta}\right)+c_{\zeta}}{e^{i \theta} f^{\prime}\left(e^{i \theta}\right)}\right\},
$$

then (a) and (b) continue to hold.

Regarding (a), a result of Hengartner and Szynal [10, Theorem 3.1] asserts that if $\zeta \in \partial K\left(\rho, f^{*}\right)$ then $\rho e^{i \theta_{1}} h^{\prime}\left(\rho e^{i \theta_{1}}\right)+c_{\zeta}$ has at most one zero which is of order one.

Proof. The Jacobian of $f$ is given by

$$
J(z)=\left[\left|z h^{\prime}(z)+c_{\zeta}\right|^{2}-\left|\left(\rho^{2} / \bar{z}\right) h^{\prime}\left(\rho^{2} / \bar{z}\right)+c_{\zeta}\right|^{2}\right] /|z|^{2}, \quad(z \in \mathbb{A}(\rho, 1)) .
$$


Since $f$ is univalent and sense-preserving, Lewy's theorem [13] yields $J(z)>$ 0 . This implies $z h^{\prime}(z)+c_{\zeta} \neq 0$ for $z \in \mathbb{A}(\rho, 1)$. By Lemma 3.3, $h$ has a continuously differentiable extension to $\mathbb{A}\left(\rho^{2}, 1\right)$ such that $h^{\prime}\left(e^{i \theta}\right) \neq 0$ and $h^{\prime}\left(\rho^{2} e^{i \theta}\right) \neq 0$ for all $\theta$. It follows that $J$ has a continuous extension to $\overline{\mathbb{A}(\rho, 1)}$ such that

$$
J\left(e^{i \theta}\right)=\left|e^{i \theta} h^{\prime}\left(e^{i \theta}\right)+c_{\zeta}\right|^{2}-\left|\rho^{2} e^{i \theta} h^{\prime}\left(\rho^{2} e^{i \theta}\right)+c_{\zeta}\right|^{2},
$$

and $J\left(e^{i \theta}\right) \geq 0$ for all $\theta$. If for some $\theta, e^{i \theta} h^{\prime}\left(e^{i \theta}\right)+c_{\zeta}=0$, then $\rho^{2} e^{i \theta} h^{\prime}\left(\rho^{2} e^{i \theta}\right)+$ $c_{\zeta}=0$, and consequently

$$
e^{i \theta} f^{\prime}\left(e^{i \theta}\right)=e^{i \theta} h^{\prime}\left(e^{i \theta}\right)-\rho^{2} e^{i \theta} h^{\prime}\left(\rho^{2} e^{i \theta}\right)=0
$$

which gives a contradiction. Hence, $e^{i \theta} h^{\prime}\left(e^{i \theta}\right)+c_{\zeta} \neq 0$ for all $\theta$.

It also follows that the dilatation of $f$ given by

$$
\omega(z)=\frac{\overline{\left(\rho^{2} / \bar{z}\right) h^{\prime}\left(\rho^{2} / \bar{z}\right)}+\overline{c_{\zeta}}}{z h^{\prime}(z)+c_{\zeta}}, \quad(z \in \mathbb{A}(\rho, 1)),
$$

extends continuously to $\mathbb{A}(\rho, 1) \cup \mathbb{T}$ such that

$$
\left|\omega\left(e^{i \theta}\right)\right|=\left|\frac{\rho^{2} e^{i \theta} h^{\prime}\left(\rho^{2} e^{i \theta}\right)+c_{\zeta}}{e^{i \theta} h^{\prime}\left(e^{i \theta}\right)+c_{\zeta}}\right| .
$$

(a) We proceed to prove (a) by contrapositivity. Suppose that $\left|\omega\left(e^{i \theta}\right)\right|<1$ for all $\theta$. Then

$$
\left|\rho^{2} e^{i \theta} h^{\prime}\left(\rho^{2} e^{i \theta}\right)+c_{\zeta}\right|<\left|e^{i \theta} h^{\prime}\left(e^{i \theta}\right)+c_{\zeta}\right| .
$$

By the compactness of $\mathbb{T}$, we can find $\delta>0$ such that

$$
\left|\rho^{2} e^{i \theta} h^{\prime}\left(\rho^{2} e^{i \theta}\right)+c_{\zeta}\right|<\left|e^{i \theta} h^{\prime}\left(e^{i \theta}\right)+c_{\zeta}\right|-\delta
$$

for all $\theta$. It follows that, for $|\eta-\zeta|<\delta \log (1 / \rho)$ and any $\theta$,

$$
\left|\rho^{2} e^{i \theta} h^{\prime}\left(\rho^{2} e^{i \theta}\right)+c_{\eta}\right|<\left|e^{i \theta} h^{\prime}\left(e^{i \theta}\right)+c_{\eta}\right|
$$

where $c_{\eta}=\left(\eta-\zeta_{0}\right) /(2 \log \rho)($ see $(5.4))$.

Suppose now that $\rho e^{i \theta} h^{\prime}\left(\rho e^{i \theta}\right)+c_{\zeta} \neq 0$ for all $\theta$. Then, in view of the above, $z h^{\prime}(z)+c_{\zeta} \neq 0$ for $z \in \overline{\mathbb{A}(\rho, 1)}$. Since $\overline{\mathbb{A}(\rho, 1)}$ is compact, there is $\sigma>0$ such that $\left|z h^{\prime}(z)+c_{\zeta}\right|>\sigma$ for $z \in \overline{\mathbb{A}(\rho, 1)}$. It follows that, for $|\eta-\zeta|<2 \sigma \log (1 / \rho)$,

$$
\left|z h^{\prime}(z)+c_{\eta}\right|>0, \quad(z \in \overline{\mathbb{A}(\rho, 1)}) .
$$

Then (4.7) and (4.8) hold for every $\eta$ satisfying

$$
|\eta-\zeta|<\tau=\min \{2 \delta \log (1 / \rho), 2 \sigma \log (1 / \rho)\} .
$$

For each such $\eta$, let

$$
f_{\eta}(z)=h(z)-h\left(\rho^{2} / \bar{z}\right)+\eta+2 c_{\eta} \log (|z| / \rho), \quad(z \in \overline{\mathbb{A}(\rho, 1)}) .
$$


Then $f_{\eta}$ is a harmonic mapping whose dilatation is given by

$$
\omega_{\eta}(z)=\frac{\overline{\left(\rho^{2} / \bar{z}\right) h^{\prime}\left(\rho^{2} / \bar{z}\right)}+\bar{c}_{\eta}}{z h^{\prime}(z)+c_{\eta}}, \quad(z \in \overline{\mathbb{A}(\rho, 1)}) .
$$

Clearly, by (4.7) and (4.8), $\omega_{\eta}$ is an analytic function that extends continuously to $\overline{\mathbb{A}(\rho, 1)}$ such that $\left|\omega_{\eta}\left(\left(e^{i \theta}\right)\right)\right|<1$ and $\left|\omega_{\eta}\left(\rho\left(e^{i \theta}\right)\right)\right|=1$ for all $\theta$. Hence, by the maximum principle, $\left|\omega_{\eta}(z)\right|<1$. This yields, because of (4.8), that the Jacobian of $f_{\eta}$ is positive in $\mathbb{A}(\rho, 1)$, and consequently $f_{\eta}$ is a univalent sense-preserving harmonic mapping. Now, by invoking Theorem 2.1 and Remark 2.2, we conclude that each $f_{\eta}: \mathbb{A}(\rho, 1) \rightarrow \mathbb{G} \backslash\{\eta\}$ is a homeomorphism. Since this holds whenever $|\eta-\zeta|<\tau, \zeta$ is an interior point of $K\left(\rho, f^{*}\right)$ and we have a contradiction. This proves (a).

(b) Suppose that $\left|\omega\left(e^{i \theta_{1}}\right)\right|=1$ for some $\theta_{1}$. Then the Möbius transformation

$$
T(z)=\frac{\rho^{2} e^{i \theta_{1}} h^{\prime}\left(\rho^{2} e^{i \theta_{1}}\right)+z}{e^{i \theta_{1}} h^{\prime}\left(e^{i \theta_{1}}\right)+z}
$$

satisfies $\left|T\left(c_{\zeta}\right)\right|=1$. Since, by (5.4), $\eta-\zeta=2\left(c_{\eta}-c_{\zeta}\right) \log \rho$, any open neighborhood of $\zeta$ contains an $\eta$ such that $\left|T\left(c_{\eta}\right)\right|>1$, or equivalently, $\omega_{\eta}\left(e^{i \theta_{1}}\right)>1$ where $\omega_{\eta}$ is as defined above. Therefore, $\eta \notin K\left(\rho, f^{*}\right)$ and $\zeta \in \partial K\left(\rho, f^{*}\right)$.

(c) Since $e^{i \theta} h^{\prime}\left(e^{i \theta}\right)+c_{\zeta} \neq 0$ for all $\theta$, using (3.1), we obtain

$$
\begin{aligned}
e^{i \theta} f^{\prime}\left(e^{i \theta}\right)= & {\left[e^{i \theta} h^{\prime}\left(e^{i \theta}\right)+c_{\zeta}\right]-\left[\rho^{2} e^{i \theta} h^{\prime}\left(\rho^{2} e^{i \theta}\right)+c_{\zeta}\right] } \\
= & {\left[e^{i \theta} h^{\prime}\left(e^{i \theta}\right)+c_{\zeta}\right] } \\
& \times\left[1-\left[\rho^{2} e^{i \theta} h^{\prime}\left(\rho^{2} e^{i \theta}\right)+c_{\zeta}\right] /\left[e^{i \theta} h^{\prime}\left(e^{i \theta}\right)+c_{\zeta}\right]\right] .
\end{aligned}
$$

Since $f^{\prime}\left(e^{i \theta}\right) \neq 0$ for all $\theta$, we obtain

$$
\frac{e^{i \theta} h^{\prime}\left(e^{i \theta}\right)+c_{\zeta}}{e^{i \theta} f^{\prime}\left(e^{i \theta}\right)}=\frac{1}{1-\left[\rho^{2} e^{i \theta} h^{\prime}\left(\rho^{2} e^{i \theta}\right)+c_{\zeta}\right] /\left[e^{i \theta} h^{\prime}\left(e^{i \theta}\right)+c_{\zeta}\right]} .
$$

This implies that

$$
2 \Re\left\{\frac{e^{i \theta} h^{\prime}\left(e^{i \theta}\right)+c_{\zeta}}{e^{i \theta} f^{\prime}\left(e^{i \theta}\right)}\right\}=1
$$

for some $\theta$ if and only if $\left|\omega\left(e^{i \theta}\right)\right|=1$; see (4.6). This proves (c).

We apply Theorem 4.2 to a function $f \in \mathcal{H}_{u}\left(\rho, f^{*}\right)$ of form (2.2). In this case, $\zeta$ is the average $\zeta_{0}$ of $f^{*}$ on $\mathbb{T}, c_{\zeta}=0, \rho e^{i \theta} h^{\prime}\left(\rho e^{i \theta}\right) \neq 0$ for all $\theta$ by Theorem 3.1(a), and $\left|\omega\left(e^{i \theta}\right)\right|=1$ for some $\theta$ if and only if $\rho^{2}\left|h^{\prime}\left(\rho^{2} e^{i \theta}\right)\right|=$ $\left|h^{\prime}\left(e^{i \theta}\right)\right|$. We conclude the following Corollary 4.1.

Corollary 4.1. Let $f \in \mathcal{H}_{u}\left(\rho, f^{*}\right)$ be of form (2.2), where $f^{*}$ is as in Theorem 4.2. Then the following statements are equivalent:

(a) $\zeta_{0} \in \partial K\left(\rho, f^{*}\right)$. 
(b) $\rho^{2}\left|h^{\prime}\left(\rho^{2} e^{i \theta}\right)\right|=\left|h^{\prime}\left(e^{i \theta}\right)\right|$ for some $\theta$.

(c) $2 \Re\left\{h^{\prime}\left(e^{i \theta}\right) / f^{\prime}\left(e^{i \theta}\right)\right\}=1$ for some $\theta$.

The arguments used in the proof of Theorem 4.2 yield at once sufficient conditions for the univalence of functions in $\mathcal{H}\left(\rho, f^{*}\right)$ where $f^{*}$ is as in Theorem 4.2 .

Theorem 4.3. Let $f \in \mathcal{H}\left(\rho, f^{*}\right)$ be of form (3.1), where $f^{*}$ be smooth as in Theorem 4.2. Then $f \in \mathcal{H}_{u}\left(\rho, f^{*}\right)$ if $z h^{\prime}(z)+c_{\zeta} \neq 0$ for $z \in \overline{\mathbb{A}(\rho, 1)}$, and if one of the following two inequalities holds for all $\theta$ :

(a) $\left|\omega\left(e^{i \theta}\right)\right| \leq 1$.

(b)

$$
2 \Re\left\{\frac{e^{i \theta} h^{\prime}\left(e^{i \theta}\right)+c_{\zeta}}{e^{i \theta} f^{\prime}\left(e^{i \theta}\right)}\right\} \geq 1 .
$$

We remark that $f^{*}$ as defined in Theorem 4.2 yields, by Lemma 3.3, $z h^{\prime}(z) \neq 0$ for $z \in \overline{\mathbb{A}(\rho, 1)}$. This makes the above sufficiency condition, $z h^{\prime}(z)+c_{\zeta} \neq 0$ for $z \in \overline{\mathbb{A}(\rho, 1)}$, easily achievable for functions $f \in \mathcal{H}\left(\rho, f^{*}\right)$ with appropriately small $c_{\zeta}$.

Finally, we prove the existence of a large family of triplets, $0<\rho<1$, $\mathbb{G}_{\rho}, f^{*}$, where $\mathbb{G}_{\rho}$ is a bounded convex domain and $f_{\rho}^{*}: \mathbb{T} \rightarrow \partial \mathbb{G}_{\rho}$ is a sensepreserving homeomorphism, such that $K\left(\rho, f^{*}\right)$ has a nonempty interior containing the average of $f^{*}$.

Theorem 4.4. Let $\Omega$ be a bounded convex domain, and let $h$ be a homeomorphism of $\overline{\mathbb{D}}$ onto $\bar{\Omega}$ that maps $\mathbb{D}$ conformally onto $\Omega$. Suppose that $h^{\prime \prime}$ is continuous on $\overline{\mathbb{D}}, h^{\prime \prime}\left(e^{i \theta}\right)$ is absolutely continuous, and

$$
\Re\left\{1+e^{i \theta} \frac{h^{\prime \prime}\left(e^{i \theta}\right)}{h^{\prime}\left(e^{i \theta}\right)}\right\}>0
$$

for all $\theta$. Then there exists $\delta>0$ such that for each $0<\rho<\delta$ we can find a bounded convex domain $\mathbb{G}_{\rho}$ such that the harmonic mapping

$$
f_{\rho}(z)=h(z)-h\left(\rho^{2} / \bar{z}\right), \quad(z \in \overline{\mathbb{A}(\rho, 1)}),
$$

satisfies the following properties:

(i) $f_{\rho}: \mathbb{T} \rightarrow \partial \mathbb{G}_{\rho}$ is a sense-preserving homeomorphism.

(ii) $f_{\rho}$ is continuously twice-differentiable on $\overline{\mathbb{A}(\rho, 1)}$.

(iii) $f_{\rho} \in \mathcal{H}_{0}\left(\rho, \mathbb{G}_{\rho}\right)$.

(iv) There is $\sigma>0$, depending on $\rho$, such that for any $|\zeta|<\sigma$ the function

$$
f_{\zeta}(z)=h(z)-h\left(\rho^{2} / \bar{z}\right)+\zeta+2 c_{\zeta} \log (|z| / \rho)
$$

belongs to $\mathcal{H}_{u}\left(\rho, \mathbb{G}_{\rho}\right)$. 
Remark 4.1. (i) Without (4.9), the hypothesis of the theorem yields the following weaker form of (4.9):

$$
\Re\left\{1+e^{i \theta} \frac{h^{\prime \prime}\left(e^{i \theta}\right)}{h^{\prime}\left(e^{i \theta}\right)}\right\} \geq 0 .
$$

To see this, observe that $z h^{\prime}(z)$ is a univalent starlike function in $\mathbb{D}$ which gives

$$
\Re\left\{1+z \frac{h^{\prime \prime}(z)}{h^{\prime}(z)}\right\}>0, \quad\left(z=r e^{i \theta} \in \mathbb{D}\right) .
$$

Now, because $h^{\prime \prime}$ extends continuously to $\overline{\mathbb{D}}$, the integral

$$
\int_{0}^{z} h^{\prime \prime}(\zeta) d \zeta, \quad(z \in \overline{\mathbb{D}})
$$

where the differentiable path of integration from 0 to $z$ lies in $\overline{\mathbb{D}}$, yields, by Cauchy's theorem, the continuous extension of $h^{\prime}(z)$ to $\overline{\mathbb{D}}$. On the other hand, since $z h^{\prime}(z)$ is univalent in $\mathbb{D}$ and maps the origin to itself, $z h^{\prime}(z) \neq 0$ for $z \in \overline{\mathbb{D}}$. Then (4.12) follows at once by letting $r \rightarrow 1$ in (4.13).

(ii) Using Kellogg and Warschawski [18, Theorem 3.6, p. 49], the hypothesis that $h^{\prime \prime}(z)$ admits a continuous extension to $\overline{\mathbb{D}}$ with absolutely continuous $h^{\prime \prime}\left(e^{i \theta}\right)$ follows if $\partial \mathbb{G}$ has a parametrization $w(t), 0 \leq t \leq 2 \pi$, whose first derivative is nonvanishing and second derivative is Lipschitz of order $\alpha$, $0<\alpha<1$.

Proof of Theorem 4.4. By the compactness of $\mathbb{T}$, there is $q>0$ such that

$$
\Re\left\{1+e^{i \theta} \frac{h^{\prime \prime}\left(e^{i \theta}\right)}{h^{\prime}\left(e^{i \theta}\right)}\right\}>q
$$

for all $\theta$. For a fixed $0<\rho<1$, let

$$
k_{\rho}(z)=h(z)-h\left(\rho^{2} z\right), \quad(z \in \overline{\mathbb{D}}) .
$$

Then $k_{\rho}$ is an analytic function in $\mathbb{D}$ with $k_{\rho}(0)=0$. We can write

$$
1+e^{i \theta} \frac{k_{\rho}^{\prime \prime}\left(e^{i \theta}\right)}{k_{\rho}^{\prime}\left(e^{i \theta}\right)}=1+e^{i \theta} \frac{h^{\prime \prime}\left(e^{i \theta}\right)}{h^{\prime}\left(e^{i \theta}\right)}+e^{i \theta} q_{\rho}\left(e^{i \theta}\right),
$$

where

$$
q_{\rho}\left(e^{i \theta}\right)=\rho^{2} e^{i \theta} \frac{h^{\prime}\left(\rho^{2} e^{i \theta}\right) h^{\prime \prime}\left(e^{i \theta}\right)-\rho^{2} h^{\prime}\left(e^{i \theta}\right) h^{\prime \prime}\left(\rho^{2} e^{i \theta}\right)}{h^{\prime}\left(e^{i \theta}\right)\left[h^{\prime}\left(e^{i \theta}\right)-\rho^{2} h^{\prime}\left(\rho^{2} e^{i \theta}\right)\right]} .
$$

Let $m_{1}=\min _{\theta}\left|h^{\prime}\left(e^{i \theta}\right)\right|, M_{1}=\max _{\theta}\left|h^{\prime}\left(e^{i \theta}\right)\right|, M_{2}=\max _{\theta}\left|h^{\prime \prime}\left(e^{i \theta}\right)\right|$, and

$$
\delta=\min \left\{\sqrt{\frac{m_{1}}{2 M_{1}}}, \frac{m_{1}}{2} \sqrt{\frac{q}{M_{1} M_{2}}}\right\} .
$$


Then for $0<\rho<\delta$, it is easy to verify that $\left|q_{\rho}\left(e^{i \theta}\right)\right|<q$ which gives $\Re q_{\rho}\left(e^{i \theta}\right)>-q$. Using (4.14) and (4.16), we obtain

$$
\Re\left\{1+e^{i \theta} \frac{k_{\rho}^{\prime \prime}\left(e^{i \theta}\right)}{k_{\rho}^{\prime}\left(e^{i \theta}\right)}\right\}>0 .
$$

Using (4.15), we conclude that $k^{\prime}$ and $k^{\prime \prime}$ extend continuously to $\overline{\mathbb{D}}$. Moreover, since $z h^{\prime}(z)$ is univalent and $0<\rho<\delta, k^{\prime}(z) \neq 0$ for $z \in \overline{\mathbb{D}}$. It follows by the maximum principle and (4.17) that

$$
\Re\left\{1+z \frac{k_{\rho}^{\prime \prime}(z)}{k_{\rho}^{\prime}(z)}\right\}>0, \quad(z \in \overline{\mathbb{D}}) .
$$

Let $\mathbb{G}_{\rho}=k_{\rho}(\mathbb{D})$. We conclude that $\mathbb{G}_{\rho}$ is a bounded convex domain, and that $k_{\rho}$ is a sense-preserving homeomorphism of $\overline{\mathbb{D}}$ onto $\overline{\mathbb{G}_{\rho}}$ that maps $\mathbb{D}$ conformally onto $\mathbb{G}_{\rho}$. Now define $f_{\rho}$ as in (4.10). Then, by $(4.15), f_{\rho}\left(e^{i \theta}\right)=$ $k_{\rho}\left(e^{i \theta}\right)$ which yields (i) and (ii). Furthermore,

$$
0=f_{\rho}\left(\rho e^{i \theta}\right)=\frac{1}{2 \pi} \int_{0}^{2 \pi} f_{\rho}\left(e^{i \theta}\right) d \theta
$$

Then (iii) follows at once from Theorem B. On the other hand, by the definition of $\delta$, we obtain

$$
\rho^{2}\left|h^{\prime}\left(\rho^{2} e^{i \theta}\right)\right|<\rho^{2} M_{1}<\delta^{2} M_{1} \leq \frac{m_{1}}{2} \leq\left|h^{\prime}\left(e^{i \theta}\right)\right| .
$$

This implies $f^{\prime}\left(e^{i \theta}\right) \neq 0$. Since $h^{\prime \prime}\left(e^{i \theta}\right)$ is absolutely continuous, $f^{\prime \prime}\left(e^{i \theta}\right)$ is also absolutely continuous. Now an application of Corollary 4.1 implies (iv). This completes the proof.

\section{Nitsche's Question Revisited.}

In this section, we determine explicitly all harmonic mappings $f \in \mathcal{H}_{u}(\rho, \mathbb{G})$ whose analytic parts extend analytically throughout $\mathbb{D}$. As a consequence, we conclude that the function $f$ defined by (1.1) is the only harmonic mapping, up to rotation, in $\mathcal{H}_{0}(\rho, \mathbb{D})$, (here $\mathbb{G}$ is taken as $\mathbb{D}$ ), of $\mathbb{A}(\rho, 1)$ onto $\mathbb{A}(0,1)$ whose analytic part is analytic in $\mathbb{D}$. This somehow justifies Nitsche's question above.

Definition 5.1. Let $f \in \mathcal{H}_{u}(\rho, \mathbb{G})$. Then, by Theorem 2.1, the unrestricted limit function of $f$ coincides with a sense-preserving quasihomeomorphism $f^{*}$ except possibly on a countable subset of $\mathbb{T}$. We call the value $\zeta_{0}$ given by (1.3) the average of $f$ on $\mathbb{T}$. Denote by $f_{0}$ the Dirichlet solution in $\mathbb{A}(\rho, 1)$ of the boundary function which coincides with $f^{*}$ on $\mathbb{T}$ and is the constant $\zeta_{0}$ on $\mathbb{T}_{\rho}$. (By virtue of Theorem $2.2, f_{0} \in \mathcal{H}_{0}(\rho, \mathbb{G})$.) We call $f_{0}$ the average associate of $f$.

The result of this section is Theorem 5.1. 
Theorem 5.1. Suppose $f \in \mathcal{H}_{u}(\rho, \mathbb{G})$ has form (1.2) with $\zeta_{0}$ the average of $f$ on $\mathbb{T}$. If $h$ is analytic in $\mathbb{D}$, then

$$
\begin{aligned}
f(z) & =\sum_{n=1}^{\infty} \frac{\lambda^{n} b_{n}}{1-\rho^{2 n}}\left[z^{n}-\left(\rho^{2} / \bar{z}\right)^{n}\right]+\zeta+2 c_{\zeta} \log (|z| / \rho) \\
& =\sum_{n=1}^{\infty} \frac{\lambda^{n} b_{n}}{1-\rho^{2 n}}\left[z^{n}-\left(\rho^{2} / \bar{z}\right)^{n}\right]+\zeta_{0}+2 c_{\zeta} \log |z|,
\end{aligned}
$$

where $b_{n}, n=1,2, \ldots$, is the $n$-th coefficient of the conformal map

$$
F(z)=\zeta_{0}+\sum_{n=1}^{\infty} b_{n} z^{n}
$$

of $\mathbb{D}$ onto $\mathbb{G}$ satisfying $F(0)=\zeta_{0}$, and

$$
c_{\zeta}=\frac{\zeta-\zeta_{0}}{2 \log \rho} .
$$

Proof. By virtue of Proposition 3.1, it suffices to prove the theorem for the average associate $f_{0}$ of $f$. Using the proposition, we write

$$
f_{0}(z)=h(z)-h\left(\rho^{2} / \bar{z}\right)+\zeta_{0}, \quad(z \in \mathbb{A}(\rho, 1)) .
$$

Since $h$ is analytic in $\mathbb{D}$, the function

$$
q(z)=h(z)-h\left(\rho^{2} z\right)+\zeta_{0}
$$

is analytic in $\mathbb{D}$, maps the origin to $\zeta_{0}$, and satisfies

$$
\lim _{|z| \rightarrow 1}\left[f_{0}(z)-q(z)\right]=\lim _{|z| \rightarrow 1}\left[h\left(\rho^{2} z\right)-h\left(\rho^{2} / \bar{z}\right)\right]=0 .
$$

This implies that $f_{0}$ and $q$ have the same cluster set at each $\xi \in \mathbb{T}$. But $C\left(f_{0}, \xi\right) \subset \partial \mathbb{G}$ for $\xi \in \mathbb{T}$. Hence, by [18, Corollary 2.10], $q$, and consequently $f_{0}$ by (5.7), has a continuous extension to $\overline{\mathbb{D}}$ that assumes every value of $\overline{\mathbb{G}}$ exactly $m$ times in $\overline{\mathbb{D}}$. It follows that $f_{0}\left(e^{i(t)}\right)=F\left(e^{i \varphi(t)}\right)$ where $\varphi$ is a continuous increasing function of $(-\infty, \infty)$ with $\varphi(t+2 \pi)=\varphi(t)+2 m \pi$. Using Theorem 2.1(v), we conclude $m=1$. This implies that $q$, like $F$, is a conformal map of $\mathbb{D}$ onto $\mathbb{G}$ with $q(0)=\zeta_{0}$. By Schwarz's lemma,

$$
q(z)=F(\lambda z)
$$

for some unimodular constant $\lambda$.

Suppose

$$
h(z)=a_{0}+\sum_{n=1}^{\infty} a_{n} z^{n}, \quad(z \in \mathbb{D}) .
$$

Then (5.3), (5.6) and (5.8) yield

$$
q(z)=\zeta_{0}+\sum_{n=1}^{\infty} a_{n}\left(1-\rho^{2 n}\right) z^{n}=\zeta_{0}+\sum_{n=1}^{\infty} \lambda^{n} b_{n} z^{n} .
$$


This gives

$$
a_{n}=\frac{\lambda^{n} b_{n}}{1-\rho^{2 n}} \quad(n=1,2, \ldots) .
$$

Using (5.5), we obtain

$$
f_{0}(z)=\zeta_{0}+\sum_{n=1}^{\infty} \frac{\lambda^{n} b_{n}}{1-\rho^{2 n}}\left[z^{n}-\left(\rho^{2} / \bar{z}\right)^{n}\right] .
$$

This completes the proof.

If $\mathbb{G}=\mathbb{D}$, then Theorem 5.1 yields Corollary 5.1 by taking

$$
F(z)=\frac{z+\zeta_{0}}{1+\bar{\zeta}_{0} z}=\zeta_{0}+\left(1-\left|\zeta_{0}\right|^{2}\right) \sum_{n=2}^{\infty}\left(-\zeta_{0}\right)^{n-1} z^{n} .
$$

Corollary 5.1. Suppose $f \in \mathcal{H}_{u}(\rho, \mathbb{D})$ has form (1.2) with $\zeta_{0}$ the average of $f$ on $\mathbb{T}$ and $h$ analytic in $\mathbb{D}$. Then there is a unimodular constant $\lambda$ such that

$$
\begin{aligned}
f(z)= & \lambda\left(1-\left|\zeta_{0}\right|^{2}\right)\left\{\frac{z-\rho^{2} / \bar{z}}{1-\rho^{2}}+\sum_{n=2}^{\infty} \frac{\left(-\lambda \zeta_{0}\right)^{n-1}}{1-\rho^{2 n}}\left[z^{n}-\left(\rho^{2} / \bar{z}\right)^{n}\right]\right\} \\
& +\zeta+2 c_{\zeta} \log (|z| / \rho), \quad(z \in \mathbb{A}(\rho, 1)) .
\end{aligned}
$$

In particular, if $\zeta_{0}=0$, then

$$
f(z)=\lambda \frac{z-\rho^{2} / \bar{z}}{1-\rho^{2}}+2 c_{\zeta} \log |z|, \quad(z \in \mathbb{A}(\rho, 1)) .
$$

\section{References}

[1] D. Bshouty and W. Hengartner, Univalent solutions of the Dirichlet problem for ring domains, Complex Variables, 21 (1993), 159-169, MR 95h:30018, Zbl 794.30014.

[2] _ Problems and conjectures for harmonic mappings, preprint, 1995.

[3] _ Naghibi-Beidokhti, p-valent harmonic mappings with finite Blaschke Dilatations, Ann. Univ. Mariae Curie-Skłodowska Sect. A, 53(2) (1999), 9-26.

[4] G. Choquet, Sur un type de transformation analytique généralisant la représentation conforme et définie au moyen de fonctions harmoniques, Bull. Sci. Math., 69(2) (1945), 156-165, MR 8,93a, Zbl 063.00851.

[5] P. Duren and W. Hengartner, Harmonic mappings of multiply connected domains, Pacific J. Math., 180(2) (1997), 201-220, MR 99f:30051, Zbl 855.30020

[6] H.G. Eggleston, Convexity, Cambridge Univ. Press, London, 1958, MR 23 \#A3228, Zbl 086.153.02.

[7] G. Hardy and W. Rogosinski, Fourier Series, Cambridge Univ. Press, London, 1950, MR 13,457b, Zbl 074.05202.

[8] W. Hengartner and G. Schober, Harmonic mappings with given dilatation, J. London Math. Soc., 33(2) (1986), 473-483, MR 87j:30037, Zbl 626.30018. 
[9] _ Univalent harmonic exterior and ring mappings, J. Math. Anal. Appl., 156 (1991), 154-171, MR 92g:31001, Zbl 731.30013.

[10] W. Hengartner and J. Szynal, Univalent harmonic mappings vanishing on the exterior boundary, Canad. J. Math., 44(2) (1992), 308-323, Zbl 761.30010.

[11] W. Kaplan, Close-to-convex schlicht functions, Michigan Math. J., 1 (1952), 169-185, MR 14,966e, Zbl 048.31101.

[12] H. Kneser, Lösung de Aufgabe 41, Jahresber Deutsch Math. Verein, 35 (1926), 123124 .

[13] H. Lewy, On the non-vanishing of the Jacobian in certain one-to-one mappings, Bull. Amer. Math. Soc., 42 (1936), 689-692, Zbl 015.15903.

[14] A. Lyzzaik, Univalence criteria for harmonic mappings in multiply connected domains, J. London Math. Soc., 58(2) (1998), 163-171, MR 99m:30034, Zbl 922.30021.

[15] _ Local properties of light harmonic mappings, Can. J. Math. Soc., 44(1) (1992), 135-153, MR 93e:30048, Zbl 910.30019.

[16] J.C.C. Nitsche, On the module of doubly-connected regions under harmonic mappings, Amer. Math. Monthly, 69 (1962), 781-782, Zbl 109.30503.

[17] _ Vorlesungen p̈ber Minimalflächen, Springer-Verlag, New York, 1975, MR 6 \#6533, Zbl 319.53003.

[18] Ch. Pommerenke, Boundary behaviour of conformal maps, Springer-Verlag, New York, 1991,MR 95b:30008, Zbl 762.30001.

[19] T. Radó, Aufgabe 41, Jahresber Deutsch Math. Verein, 35 (1926), 49.

[20] T. Sheil-Small, On the Fourier series of a finitely described convex curve and a conjecture of H.S. Shapiro, Math. Proc. Camb. Phil. Soc., 98 (1985), 513-527, MR 86m:42009, Zbl 602.30023.

[21] M. Tsuji, Potential theory in modern function theory, (second edition), Chelsea Publishing Company, New York, 1975, MR 54 \#2990, Zbl 322.30001.

Received October 15, 1999.

Department of Mathematics

AMERICAN UNIVERSity OF BeIRUT

BEIRUT

LEBANON

E-mail address: lyzzaik@aub.edu.lb 